\title{
Theoretical approaches to superionic conductivity ${ }^{\dagger}$
}

\author{
C S SUNANDANA* and P SENTHIL KUMAR \\ School of Physics, University of Hyderabad, Hyderabad 500 046, India \\ ${ }^{\$}$ Present Address: Physical Chemistry Division, National Chemical Laboratory, Pune 411 008, India
}

MS received 12 June 2003; revised 27 October 2003

\begin{abstract}
Recent theoretical approaches to the understanding of superionic conductivity in polycrystalline, glassy and polymeric materials are briefly reviewed. Phase transitions to the superionic conducting state in the AgI family are apparently triggered by cluster formation and strong mobile ion interaction within the clusters. Anomalous conductivity and related physical properties are explained in the cluster induced distortion model. Ionic composites such as $\mathrm{AgX}: \mathrm{Al}_{2} \mathrm{O}_{3}(X=\mathrm{Cl}, \mathrm{Br}$ and $\mathrm{I})$ involve conducting and non-conducting phases and the all-important interface between the two whose space charge enhances the conductivity and also trigger phase transitions to exotic polymorphic phases, for which the mechanisms are yet to be explored. Ion hopping dynamics controls the conductivity of superionic glasses. Mode coupling and jump relaxation theories account for the non-Debye relaxation observed in a.c. conductivity of these glasses. The theory of conductivity in polymer electrolytes-still in its infancy-involves their complex structure and glass transition behaviour. Preparative and thermal history, composition and crystallinity control ionic conductivity. New approaches to the synthesis of optimal polymer electrolytes such as rubbery electrolytes, crystalline polymers and nanocomposites must be considered before achieving a comprehensive theoretical understanding.
\end{abstract}

Keywords. Superionic conductors; theory of ionic conductivity; glasses; composites; polymer electrolytes.

\section{Introduction}

Ion motion in solids is a very interesting and challenging problem in condensed matter science. Solids come in a variety of structures and forms: single crystalline, polycrystalline (several polymorphs are possible) and nanocrystalline, glassy and polymeric. Accordingly, the potential landscape in which the ions move changes remarkably across this gamut. Even within a given solid, temperature and excitation frequencies can trigger a whole range of ion dynamic phenomena. Furthermore, the frequency dependent conductivity of several of these complex systems exhibits universal behaviour. The main experimental probe of conductivity spectroscopy along with probes such as chemical diffusion, magnetic resonance and relaxation, light, X-ray and neutron scattering as well as thermal probes such as heat capacity and thermal expansion have helped unravel the secrets of ion dynamics in these materials with the help of suitable theoretical approaches (Salamon 1975; Suresh Chandra 1981; Sunandana 1998). We shall focus on a few of these approaches as they relate to representative superionic conducting systems.

\footnotetext{
*Author for correspondence

${ }^{\dagger}$ Based on an invited talk given by the first author at the National Workshop on Solid State Ionics and its Applications, Bharatiar University, Coimbatore, 18-23 January 2002.
}

The superionic state is the penultimate stage in the saga of the 'perfect crystal' (Maier 2000). Thermodynamics and statistical mechanics decide the fate of the perfect crystal that is destined to go through a number of 'imperfect' states on its way to the molten state as the defect densities increase progressively (figure 1). The real ionic solid is essentially a defect solid and a superionic is more so. Superionic conductivity i.e. $\sigma \sim 10^{-2} \mathrm{~S} / \mathrm{cm}$ at temperatures close to room temperature is exhibited by the first three of the following four major classes of compounds which have been investigated for their temperature dependant physical properties including structural, thermal and magnetic properties. (i) $\mathrm{AgX}$ family (AgI, $\mathrm{AgBr}, \mathrm{CuBr}$ and $\mathrm{CuI}$ ) including $\mathrm{Ag}_{3} \mathrm{SBr}, \mathrm{Ag}_{3} \mathrm{SI}$ and $\mathrm{RbAg}_{4} \mathrm{I}_{5}$, (ii) $\mathrm{MX}-\mathrm{Al}_{2} \mathrm{O}_{3}$ composites ( $\mathrm{MX}=\mathrm{AgI}, \mathrm{AgCl}$, LiI etc), (iii) glasses in the system $\mathrm{AgI}-\mathrm{Ag}_{2} \mathrm{O}-\mathrm{M}_{x} \mathrm{O}_{y} \quad(\mathrm{M}=\mathrm{B}$, $x=2, y=3 ; \mathrm{M}=\mathrm{Te}, x=1, y=2$ etc) and (iv) polymeric systems such as $\mathrm{LiClO}_{4}$. polyethylene oxide. Nanocrystalline phases occur in families (ii)-(iv) during the processing stage itself and introduce mesoscopic effects.

Two major questions concerning theoretical understanding of experimental results on these materials are (i) what is the mechanism of ion transport in each of these systems? and (ii) how does the property of ion conducting relate to other physical properties? The motivation for the study of these compounds is their challenging physics and application potential in the areas of secondary batteries, electrochemical sensors, fuel cells and electrochromic devices. 


\subsection{Basics of $\sigma_{i}$}

Ionic conductivity, $\sigma_{\mathrm{i}}$, arises when an electric field, $E$, is applied to an ionic solid. A current density, $J$, developed in the material is given by

$$
J=\sigma_{\mathrm{i}} E .
$$

$\sigma_{\mathrm{i}}$ is related to mobile ion density $N$, the mobile ion charge $(\mathrm{Ze})$ and the velocity of the mobile ion per unit electric field or ion mobility, $\mu$.

$$
\sigma_{\mathrm{i}}=N(Z e) \mu .
$$

Ion mobility changes with temperature as

$$
\mu=\mu_{0} \exp \left(-E_{\mathrm{a}} / k T\right)
$$

Thus

$$
\sigma=\sigma_{0} \exp \left(-E_{\mathrm{a}} / k T\right)
$$

where

$$
\sigma_{0}=N Z e \mu_{0} .
$$

$E_{\mathrm{a}}$ is the electrostatic energy barrier that the ion has to overcome in order to hop from one site to another in the crystal/material. Ionic conductivity of a crystal is zero at absolute zero. A non-zero ionic conductivity at a non-zero temperature is the result of defects in a crystal. These defects may be thermodynamic as in NaI or 'statistical' as in AgI (figure 2). The term 'statistical' comes from strong interaction between structural defects arising due to their large concentration. NaI has isolated non-interacting Schottky defects (i.e. isolated $\mathrm{Na}^{+}$and $\mathrm{I}^{-}$vacancies), while AgI has Frenkel defects $\left(\mathrm{Ag}^{+}\right.$vacancies and $\mathrm{Ag}^{+}$interstitials) that interact considerably. Note that the conductivity of an ionic/superionic conductor increases exponentially with temperature. Exactly opposite is the case of metallic conductivity - which decreases with increasing temperature. Three mechanisms (a) vacancy jump, (b) interstitial jump and (3) interstitialcy (Lidiard 1957) are possible for ionic conduction (figure 3 ). In the following sections, theoretical approaches to the four class of materials mentioned above are briefly discussed.

\section{Phase transitions and conductivity in MX systems}

To realize superionic conductivity it is necessary to increase the number of intrinsic defects and to lower the activation energy for ion jump in an ionic material. AgI is an example where both these criteria are naturally fulfilled. $\mathrm{AgI}$ exhibits polymorphism like $\mathrm{SiC}$ or $\mathrm{NbSe}_{2}, \mathrm{TaS}_{2}$. It has a stable hexagonal structure or a metastable zincblende structure (Patnaik and Sunandana 1998), both of

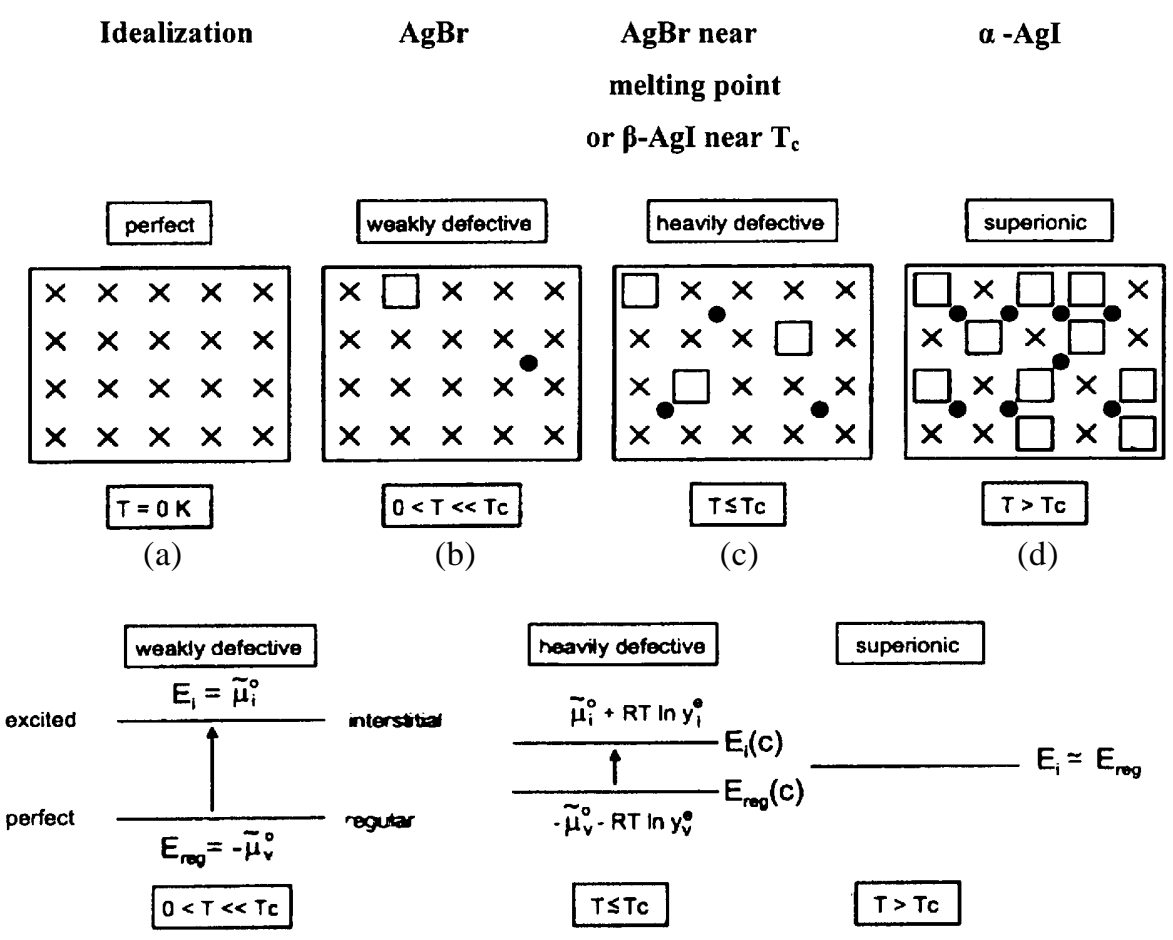

Figure 1. Progressive evolution of a crystal. (a) Ideal crystal with zero defects-an ionic insulator, (b) real crystal with non-interacting defects-an ionic conductor, (c) real crystal with large concentration of mobile ions and (d) an even large concentration of interactive defects after a phase transition-a superionic conductor. The lower part of the figure gives the energy level description of the perfect, real and superionic conductors. Note temperature dependence of chemical potential $\mu$ (from Maier 2000). 
which support Frenkel defects i.e. $\mathrm{Ag}^{+}$vacancies and $\mathrm{Ag}^{+}$ interstitials. The family of AgI-type superionic conductors may be considered as I-VII semiconductors. Figure 4 displays the temperature dependencies of electrical conductivity (Kvist and Josefson 1968), lattice parameters (Takahashi 1987; Ishii and Kamishima 1999), heat capacity (Nolting and Rein 1969), thermal expansion (Senthil Kumar et al 2000); and magnetic susceptibility (Takahashi 1987). We can immediately infer that all these properties along with conductivity exhibit an anomaly around $147^{\circ} \mathrm{C}$. At this temperature, (i) conductivity changes abruptly and discontinuously by $\sim 4$ orders of magnitude, (ii) the hexagonal (wurtzite type) lattice sud- denly collapses into a body centred cubic lattice, (iii) heat capacity shows a jump characteristic of an order-disorder type phase transition, (iv) thermal expansion coefficient $(\alpha)$, which is fairly constant but slightly below zero (negative), shows a sharp negative anomaly at the transition temperature and (v) the diamagnetic susceptibility shows a jump but stabilizes to a less negative value thereafter.

These results show that the transition to the high conductivity state is first order type. How does one explain the conductivity transition in AgI? Let us follow the cluster-distortion coupling model of Ishii and Kamishima (1999). This is a realistic statistical model that considers interaction between ion clusters rather than individual
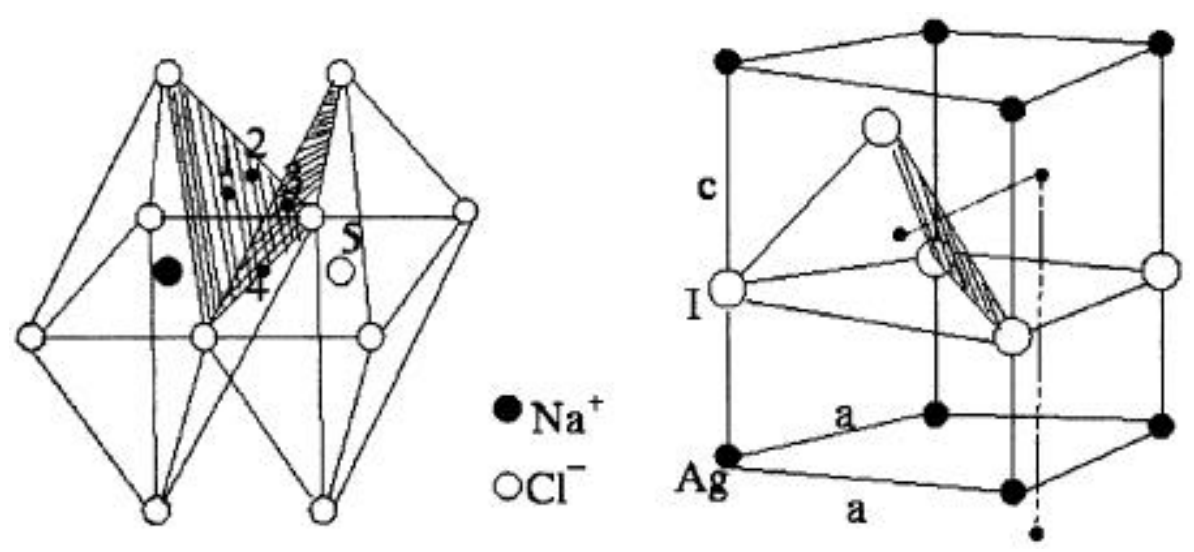

Figure 2. NaI (left) has stable, 'closed' rocksalt structure with 'thermodynamic' Schottky defects while AgI (right) has 'open' wurtzite structure 'stabilized' cationic $\left(\mathrm{Ag}^{+}\right)$ Frenkel defects, which is stable only up to $147^{\circ} \mathrm{C}$.

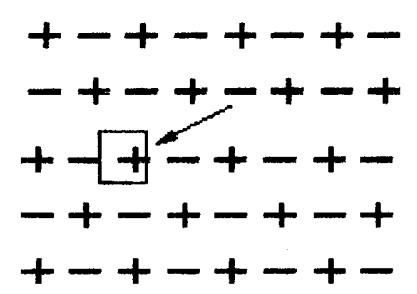

(a) Vacancy mechanism

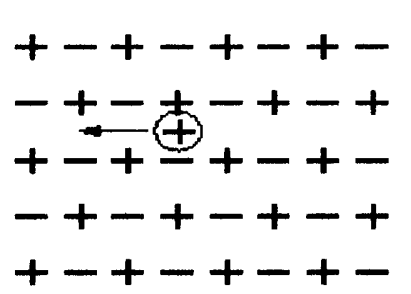

(b) Intersitial mechanism

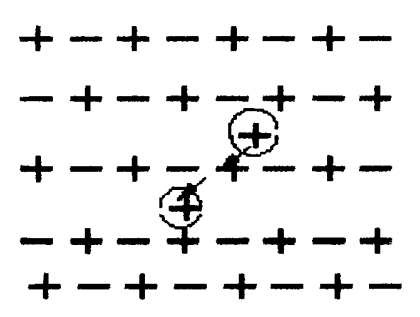

\section{(c) Interstitialcy mechanism}

Figure 3. The three basic mechanisms for ionic conduction in crystals involving the motion of vacancies and interstitials. 

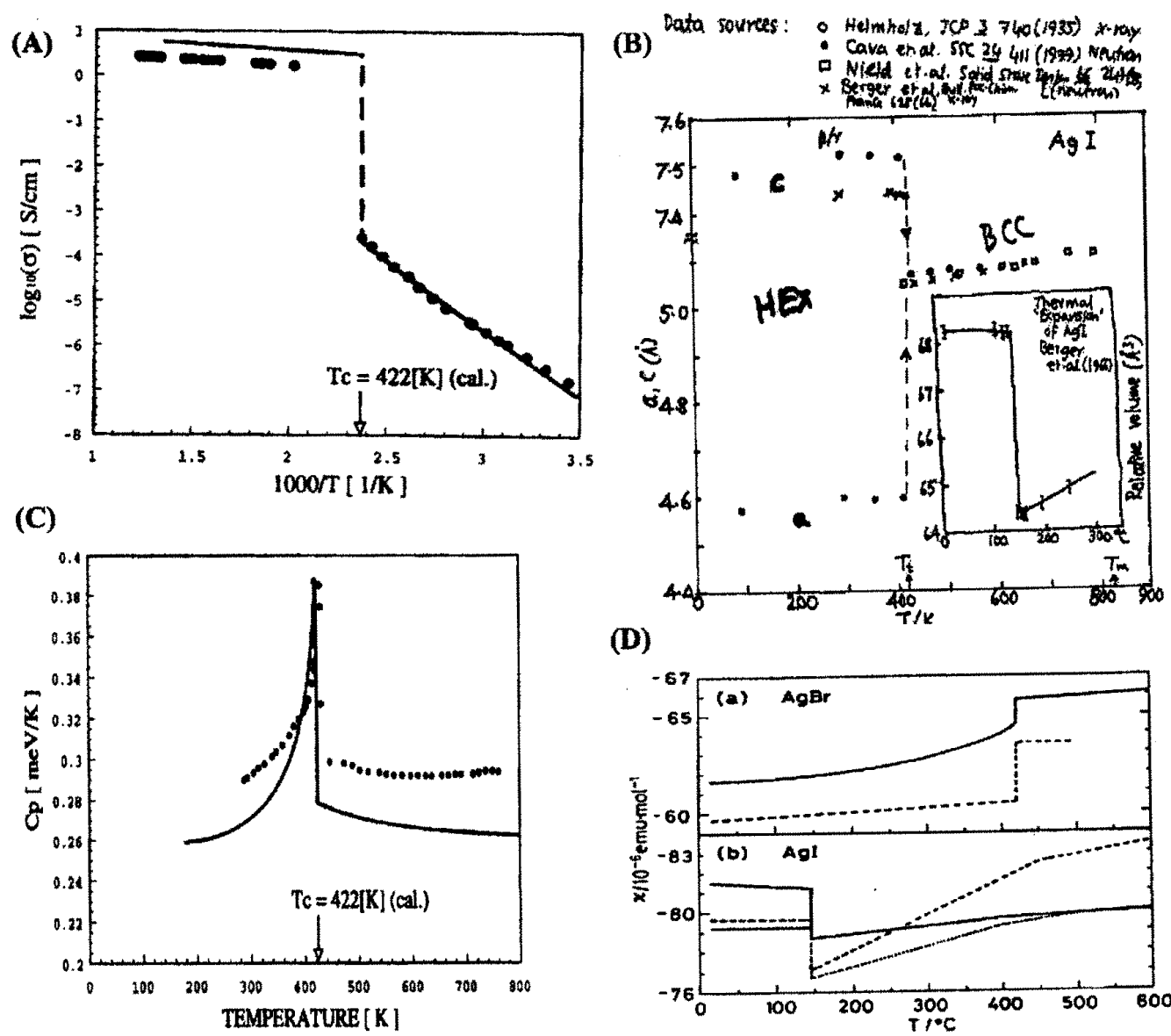

(D)

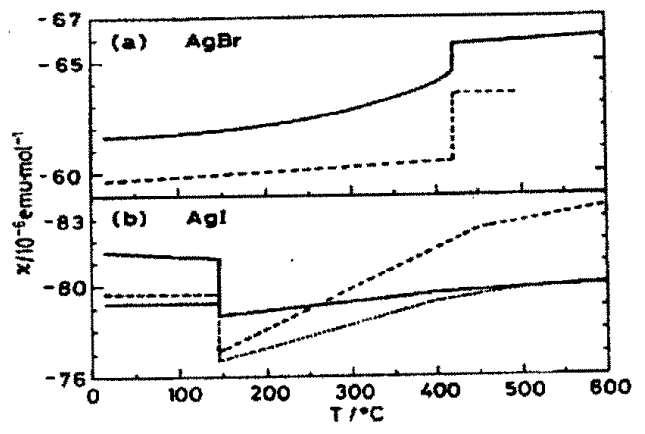

Figure 4. Basic physical properties of $\mathrm{AgI}$ as functions of temperature. (A) Ionic conductivity (experimental points and fit to cluster-induced distortion model), (B) lattice parameters and thermal expansion (inset, refs.), (C) heat capacity (exptl. points and theoretical fit) and (D) diamagnetic susceptibility of $\mathrm{AgBr}$ and $\mathrm{AgI}$.

ions in the spirit of a lattice gas approximation for the mobile ions.

In this model, primitive cells consisting of two species of ions, immobile $\left(\mathrm{I}^{-}\right)$and mobile $\left(\mathrm{Ag}^{+}\right)$, are considered. At ground state, in the low temperature $\left(T<147^{\circ} \mathrm{C}\right)$ phase, $\mathrm{Ag}^{+}$ion occupies A-site (A-sublattice) and $\mathrm{I}^{-}$ion occupies $\mathrm{C}$-site (C-sublattice). A third sublattice is $\mathrm{B}$, which is empty at the ground state (figure 5). At high temperatures, a fraction of the mobile $\mathrm{Ag}^{+}$ions can be excited into the B-sublattice. These together with the ions of Asublattice form clusters. For example, $\mathrm{Ag}^{+}$at $\mathrm{A}$-site gets excited to its adjacent B-site. Then, $\mathrm{I}^{-}$at the B-site's nearest neighbour $\mathrm{C}$, distorts towards $\mathrm{B}$, due to the excess charge of new clusters. This is the cluster induced local distortion. The Hamiltonian for the problem is,

$$
H=H_{\mathrm{LG}}+H_{\mathrm{SD}}+H_{\mathrm{vib}}+H_{\mathrm{int}}
$$

where $H_{\mathrm{LG}}$ is the so called 'lattice gas' term for mobile ions, $H_{\mathrm{SD}}$ the static distortion (elastic energy stored by cluster induced distortion), $H_{\text {vib }}$ the vibrational part corresponding to the anharmonic optical phonons (note that

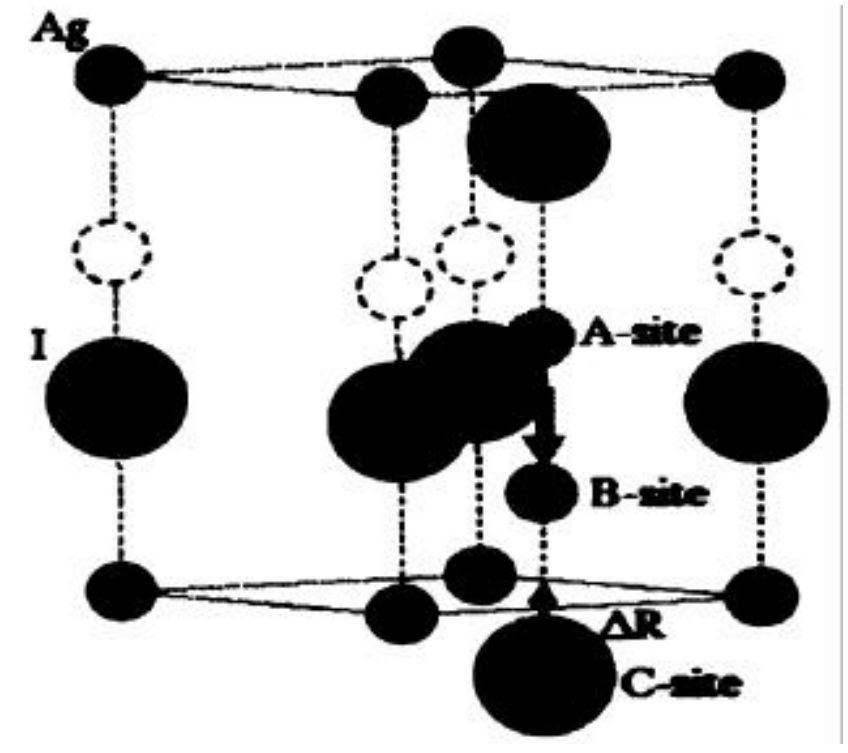

Figure 5. The $\mathrm{A}\left(\mathrm{Ag}^{+}\right.$-based), $\mathrm{B}$ (empty) and $\mathrm{C}$ ( $\mathrm{I}^{-}$based) sublattices for AgI in the cluster induced distortion model. The B sublattice is progressively occupied on heating (Ishii and Kamishima 1999). 
the pre-exponential factor in conductivity contains an attempt frequency of the order of the frequency of optical phonons). $H_{\text {int }}$ represents the interaction between nearest neighbour pair clusters and distortion. Using this $H$, the free energy of the primitive cell $\left(f_{\text {cell }}\right)$ and its temperature dependence are calculated. The free energy of the system gets lowered due to the clustering of mobile ions. The system tends to produce a cluster of Ag ions. This triggers a phase transition at a temperature of $422 \mathrm{~K}$ according to calculations in the cluster-induced distortion-coupling model. The order parameter for the transition is the difference in the $\mathrm{Ag}^{+}$occupation probability in $\mathrm{A}$ and $\mathrm{B}$ sublattices. This calculation exactly reproduces the temperature dependent d.c. conductivity profile of AgI and $\mathrm{CuBr}$. It is obtained by the pair approximation of the path probability method. The final expression for $\sigma$ of $\mathrm{AgI}$ is given by,

$$
\begin{aligned}
\sigma_{\mathrm{AgI}}= & \sigma_{0}\{1+[2((1 /(1+\xi-2 y))-(1 /(1-\xi-2 y)))+ \\
& \left.\left.(\exp (\beta \varepsilon)-1)\left(\left(1 / \Lambda^{\mathrm{A}}\right)-\left(1 / \Lambda^{\mathrm{B}}\right)\right)\right] \Delta y\right\}, \\
\sigma_{0}= & \beta m_{\mathrm{L}}(e l)^{2} \Gamma(1-\xi-2 y)\left\{2 \Lambda^{\mathrm{B}} /(1-\xi)\right\}^{2 \gamma-1}, \\
& \Gamma=\omega_{\mathrm{s}} \exp (-\beta U), \\
\Lambda^{\mathrm{A}}= & (\exp (\beta \varepsilon)-1) y+(1 / 2)(1+\xi), \Lambda^{\mathrm{B}}= \\
& (\exp (\beta \varepsilon)-1) y+(1 / 2)(1-\xi), \\
\Delta y= & (1 / A)\{(1-\xi-2 y) /(1-\xi)- \\
& (1+\xi-2 y) /(1+\xi)\}, \\
A= & {[(1-\xi-2 y) /(1-\xi)]\{-[(4 /(1-\xi-2 y))+} \\
& (2 /(1+\xi-2 y))+(3 / y)]+(\exp (\beta \varepsilon)-1)\left(\left(2 / \Lambda^{\mathrm{A}}\right)+\right. \\
& \left.\left.\left(1 / \Lambda^{\mathrm{B}}\right)\right)\right\}+[(1+\xi-2 y) /(1+\xi)]\{-[(4 /(1+\xi-2 y))+ \\
& (2 /(1-\xi-2 y))+(3 / y)]+(\exp (\beta \varepsilon)-1) \\
& \left.\left(\left(2 / \Lambda^{\mathrm{B}}\right)+\left(1 / \Lambda^{\mathrm{A}}\right)\right)\right\},
\end{aligned}
$$

where $\varepsilon$ is the interaction energy, $\xi$ the order parameter and $y$ the pair probability, and, for $\mathrm{CuBr}$,

$$
\begin{aligned}
\sigma_{\mathrm{CuBr}}= & \sigma(\infty)\{1+[2((1 /(1+\xi-2 y))-(1 /(1-\xi-2 y)))+ \\
& \left.\left.(\exp (\beta \varepsilon)-1)\left(\left(1 / \Lambda^{\mathrm{A}}\right)-\left(1 / \Lambda^{\mathrm{B}}\right)\right)\right] \Delta y\right\}, \\
\sigma(\infty)= & (3 / 2) \beta m_{\mathrm{L}}(e a)^{2} \Gamma(1-\xi-2 y)\left\{2 \Lambda^{\mathrm{B}} /(1-\xi)\right\}^{2 \gamma-1}, \\
& \Gamma=\omega_{\mathrm{s}} \exp (-\beta U), \\
\Lambda^{\mathrm{A}}= & (\exp (\beta \varepsilon)-1) y+(1 / 2)(1+\xi), \\
& \Lambda^{\mathrm{B}}=(\exp (\beta \varepsilon)-1) y+(1 / 2)(1-\xi), \\
\Delta y= & (1 / A)\{(1-\xi-2 y) /(1-\xi)- \\
& (1+\xi-2 y) /(1+\xi)\}, \\
A= & (1-\xi-2 y) /(1-\xi)]\{-[(8 /(1-\xi-2 y))+ \\
& (2 /(1+\xi-2 y))+(5 / y)]+(\exp (\beta \varepsilon)-1) \\
& \left.\left(\left(4 / \Lambda^{\mathrm{A}}\right)+\left(1 / \Lambda^{\mathrm{B}}\right)\right)\right\}+[(1+\xi-2 y) /(1+\xi)] \\
& \{-[(8 /(1+\xi-2 y))+(2 /(1-\xi-2 y))+(5 / y)]+ \\
& \left.(\exp (\beta \varepsilon)-1)\left(\left(4 / \Lambda^{\mathrm{B}}\right)+\left(1 / \Lambda^{\mathrm{A}}\right)\right)\right\} .
\end{aligned}
$$

The excess specific heat,

$$
\begin{aligned}
& \Delta C_{\mathrm{p}}=-(1 / 2) T\left(\partial^{2} f_{\text {pair }} / \partial T^{2}\right)_{\mathrm{p}} \text { for } \mathrm{CuBr}, \\
& \Delta C_{\mathrm{p}}=-(1 / 2) T\left(\partial^{2} f_{\text {cell }} / \partial T^{2}\right)_{\mathrm{p}}+(1 / 2)(6-1) k_{\mathrm{B}} \text { for } \mathrm{AgI},
\end{aligned}
$$

( $f_{\text {pair }}$ is the free energy of a pair of $\mathrm{Cu}$ and $\mathrm{Br}$ ) observed at the phase transition is also accounted for in this model. The negative thermal expansion is explained in terms of the static local distortion becoming larger than the dynamic distortion. This is because a large number of $\mathrm{Ag}^{+}$ vacancies are excited just below the transition temperature.

Application of this model to $\mathrm{Cu}$-rich $\mathrm{CuI}-\mathrm{AgI}$ solid solutions as well as $\mathrm{Ag}$-rich $\mathrm{AgBr}-\mathrm{CuBr}$ solid solutions should be possible. The case of $\mathrm{CuI}$ might need a substantial modification.

AgI is a diamagnetic material and its diamagnetic susceptibility arises (Takahashi 1987) from three contributions (in the band orbital model) viz. $\chi_{c}$ from core electrons, $\chi_{\mathrm{v}}$ from valence electrons and $\chi_{\mathrm{p}}$ from VanVleck paramagnetism. $\chi_{\mathrm{c}}$ and $\chi_{\mathrm{v}}$ are negative while $\chi_{\mathrm{p}}$ is positive.

$$
\begin{aligned}
& \chi=\chi_{\mathrm{c}}+\chi_{\mathrm{v}}+\chi_{\mathrm{p}}, \\
& \mathrm{d} \chi / \mathrm{d} T=\left(\mathrm{d} \chi_{\mathrm{v}} / \mathrm{d} T\right)+\left(\mathrm{d} \chi_{\mathrm{p}} / \mathrm{d} T\right)
\end{aligned}
$$

For partially covalent bonded materials, $\chi_{\mathrm{v}} \gg \chi_{\mathrm{p}} \cdot \chi_{\mathrm{p}}$ reflects the local symmetry of the neighbours and thus $\left(\mathrm{d} \chi_{\mathrm{p}} / \mathrm{d} T\right)<\left(\mathrm{d} \chi_{\mathrm{v}} / \mathrm{d} T\right)$ for the same phase. Therefore,

$$
\chi_{\mathrm{v}}=(1 / 2 \alpha)(\mathrm{d} \chi / \mathrm{d} T)
$$

where $\alpha$ is the thermal expansion coefficient. Then,

$$
(\mathrm{d} \chi / \mathrm{d} T)=\left(\mathrm{d} \chi_{\mathrm{v}} / \mathrm{d} T\right) .
$$

How is this related to ionic conductivity? There is a correlation between $(1 / \chi)(\mathrm{d} \chi / \mathrm{d} T)$ and the ionicity in $\mathrm{A}^{N} \mathrm{~B}^{8-N}$ compounds. For the AgI group of compounds, $N=1$. For $\alpha$-AgI, there is a deviation from this correlation due to a possible contribution of $d$-electrons to the valence state.

The iono-covalent nature of the Ag-I bond is invoked in an electronic theory of superionic conductors (Aniya 2000b). When AgI is heated, local changes occur in Ag-I bonding and these changes play a leading role in the phase transition to the superionic state. Local fluctuations of bonding create field of forces that cause ion movement. The transition to the superionic state occurs when the bond fluctuations go critical resulting in a collapse of the $\mathrm{Ag}^{+}$sublattice. Lattice vibrations induce overlap of $4 d$-electron wavefunctions ( $\mathrm{Ag}$ ) with the $5 p$-wavefunctions of $\mathrm{I}^{-}$from time to time. This time varying degrees of participation of $d$-electrons in Ag-I bond leads to fluctuations. Therefore, bond fluctuations essentially mean temperature/time dependent local bond lengths. And when these go critical, 'sublattice melting', a phenomenon akin to attainment of thermodynamic liquid state from 
the solid state, occurs. In fact, Lindemann theory of melting has been applied to this phase transition to estimate the transition temperature. Lindemann criterion is

$$
T_{\mathrm{c}}=\left[\mu k_{\mathrm{B}} \theta_{\mathrm{D}}^{2} d_{0}^{2} / 9 \hbar^{2}\right] x^{2}
$$

where $\mu$ is the reduced mass, $k_{\mathrm{B}}$ the Boltzmann constant, $\theta_{\mathrm{D}}$ the Debye temperature, $d_{0}$ the equilibrium interatomic distance and $x=\Delta d / d_{0}$, where $\Delta d$ is the mean square amplitude of vibration of ions around their equilibrium position at the transition temperature. $\Delta d$ is related to the highest bonding orbital energy, $\Delta E$ (beyond which the Ag-I bond collapses and sublattice melting occurs). This energy depends on the interatomic distance. Experimental $T_{\mathrm{c}}$ of AgI is reproduced for $\Delta E=0.07 \mathrm{eV}$ and $x=0.055$.

The $\beta-\alpha$ phase transition in AgI has been interpreted in a plasma model (Aniya and Wakamura 1998) in terms of the temperature dependence of the effective number of valence electrons $(Z) . Z$ shows an abrupt decrease at the transition temperature. In the plasma model, the band gap $\left(E_{\mathrm{g}}\right)$ of $\mathrm{AgI}$ is written as the energy difference between the plasmon energies of $\mathrm{AgI}$ and $\mathrm{Ag}$ as,

$$
E_{\mathrm{g}}=\left(\hbar \omega_{\mathrm{p}}\right)_{\mathrm{AgI}}-\left(\hbar \omega_{\mathrm{p}}\right)_{\mathrm{Ag}} .
$$

This is based on the collective excitation studies on insulators. Plasmon energy,

$$
\hbar \omega_{\mathrm{p}}=\hbar\left(4 \pi N e^{2} / m\right)^{1 / 2},
$$

where $N$ is the effective number of valence electrons and $m$ the electron mass.

$$
N=Z \rho N_{\mathrm{A}} / w
$$

where $Z$ is the effective number of valence electrons per formula unit, $\rho$ the density, $N_{\mathrm{A}}$ the Avagadro's number and $w$ the molecular weight. Then,

$$
\hbar \omega_{\mathrm{p}}=28 \cdot 8(Z \rho / w)^{1 / 2} \mathrm{eV} .
$$

From the knowledge of the band gap, $Z$ the number of valence electrons involved in bonding can be obtained. This is probably the most direct demonstration of the role of electronic band structure, particularly the $4 d$ orbital of $\mathrm{Ag}$, in triggering the superionic phase transition. It would be interesting to examine phase transitions in $\mathrm{CuBr}$ and $\mathrm{CuI}$ in this picture, which connects to the bond fluctuation model discussed earlier.

These theoretical approaches thus provide answers to the questions raised in the introduction, as far as AgI family is concerned-which includes $\mathrm{CuBr}, \mathrm{CuI}$ and also $\mathrm{Ag}_{3} \mathrm{SBr}, \mathrm{Ag}_{3} \mathrm{SI}$ and $\mathrm{MAg}_{4} \mathrm{I}_{5}\left(\mathrm{M}=\mathrm{K}, \mathrm{Rb}\right.$ and $\left.\mathrm{NH}_{4}\right)$ and also $\mathrm{KCu}_{4} \mathrm{I}_{5}$, which have large intrinsic $\mathrm{Ag}^{+}$(or $\mathrm{Cu}^{+}$) conductivity through a low/moderately high temperature phase transition. The role of $3 d / 4 d$ bond in stabilizing a weakly covalent tetrahedral bond and an open structure results in the rich phenomenology reported above. The exact origin of the first order phase transition and its kinetics are still open problems.

\section{The case of composites}

In composites, one attempts to enhance the conductivity of a normal ionic conductor, $\mathrm{MX}$ (e.g. $\mathrm{AgCl}$, LiI) by mixing it with an insulator (e.g. $\mathrm{Al}_{2} \mathrm{O}_{3}$ ) (Maier 1985; Laskar 1992; Shukla and Sharma 1992; Vidyullatha and Sunandana 1993). The conductivity of the composite (MX-A) is governed by ionic transport via the MX-A interface. It is determined by (a) conductivity of MX within the interface region, (b) the effective thickness of the interface region and (c) the grain size and shape of the insulating particles. Two models are of interest: (i) the percolating random resistor model and (ii) the brick wall model.

In the first model, a network of randomly connected resistors involving the non-conducting particles is imagined. Conduction takes place by 'percolation' of the electrolyte through the 'maze' of random resistors.

The 'brick wall model' regards the composite to be made of mainly bricks, composed of non-conducting particles. The 'walls' are the surfaces of these bricks important for conductivity as the electrolyte meets the bricks at these walls. The model gives the volume fraction of the interface, $f_{\mathrm{s}}$ as

$$
f_{\mathrm{s}}=\left(2 \beta \lambda / r_{\mathrm{A}}\right) \cdot f \cdot(1-f),
$$

where $\beta$ is the geometric factor $(=3), \lambda$ the thickness of the interface layer (between $\mathrm{AgCl} / \mathrm{LiI}$ and $\mathrm{Al}_{2} \mathrm{O}_{3}$ ), $r_{\mathrm{A}}$ the grain size of the dispersoid (i.e. $\mathrm{Al}_{2} \mathrm{O}_{3}$ ) and $f$ the volume fraction of the insulating phase. A preliminary study of AgBr : $x \mathrm{ZrO}_{2}(x=10,15$ and $20 \%)$ by conductivity and dielectric constant analysis (Lee et al 2000) has detected $\sim 1$ order enhancement of conductivity with respect to $\mathrm{AgBr}$. The formation of a new kinetic path along the interface is probably responsible for conductivity enhancement.

A general, easy-to-apply mixing model (Uvarov 2000) can be used to estimate the conductivity of the composite. The composite is made up of three phases, MX, A and $S$, the interface between the two. For the three-phase mixing,

$$
\sigma^{\alpha(f)}=\left(1-f-f_{\mathrm{s}}\right) \cdot \sigma_{\mathrm{MX}}^{\alpha(f)}+f_{\mathrm{s}} \cdot \sigma_{\mathrm{s}}^{\alpha(f)}+f \cdot \sigma_{\mathrm{A}}^{\alpha(f)} .
$$

The general mixing rule for a composite solid electrolyte is given by

$$
\begin{aligned}
& \sigma^{\alpha 1 \cdot(1-f)+\alpha 2 \cdot f}=\left[1-f-\left(2 \beta \lambda / r_{\mathrm{A}}\right) \cdot f \cdot(1-f)\right] \\
& \sigma_{\mathrm{MX}}^{\alpha 1 \cdot(1-f)+\alpha 2 \cdot f}+\left(2 \beta \lambda / r_{\mathrm{A}}\right) \cdot f \cdot(1-f) \text {. } \\
& \sigma_{\mathrm{S}}^{\alpha 1 \cdot(1-f)+\alpha 2 \cdot f}+f \cdot \sigma_{\mathrm{A}}^{\alpha 1 \cdot(1-f)+\alpha 2 \cdot f},
\end{aligned}
$$

where $\sigma_{\mathrm{MX}}$ and $\sigma_{\mathrm{S}}$ are the conductivity of MX in the bulk and within the interface region, respectively and $\sigma_{\mathrm{A}}$ the conductivity of the insulator. The superscript, $\alpha$, refers to a conductivity parameter describing the arrangement of components in the composite. For the case, where $\sigma_{\mathrm{S}} \gg \sigma_{\mathrm{MX}}$,

$$
\sigma=\sigma_{\mathrm{S}} \cdot\left[\beta \cdot\left(2 \lambda / r_{\mathrm{A}}\right) \cdot f \cdot(1-f)\right]^{1 /[\alpha 1 \cdot(1-f)+\alpha 2 \cdot f]}
$$


Ideal parallel connectivity (with respect to current direction) implies $\alpha=L$. For serial connectivity, $\alpha=-1$ so that $\alpha$ can be obtained from the morphology of the composite.

$\mathrm{AgX}: \mathrm{Al}_{2} \mathrm{O}_{3} \quad(\mathrm{X}=\mathrm{Cl}, \mathrm{Br}$ and $\mathrm{I})$ composites exhibit enhanced conductivity due to (a) ideal space charge effects in the case of $\mathrm{AgCl}$ and $\mathrm{AgBr}$ and (b) interfacial phase transition in the case of AgI. The space charge effects are the result of $\mathrm{Ag}^{+}$adsorption to the basic $\mathrm{Al}_{2} \mathrm{O}_{3}$ surfaces.

$\mathrm{AgCl}$ and $\mathrm{AgBr}$ have robust rocksalt structures. Therefore, the interaction of $\mathrm{Ag}^{+}$with nucleophilic alumina leads to an ideal diffuse and semi-infinite compensating space charge layer. $\beta$-AgI has a wurtzite structure and for a composite of $\beta$-AgI with $>30$ mol. $\% \quad \gamma-\mathrm{Al}_{2} \mathrm{O}_{3}$ an interfacial phase transition from $\beta$-AgI (or $2 \mathrm{H}$-AgI) to $7 \mathrm{H}$-AgI has been detected at the $\beta-\mathrm{AgI} / \gamma \mathrm{Al}_{2} \mathrm{O}_{3}$ interface, by means of XRD, conductivity and DSC. The unique seven-layer polytype in $\mathrm{AgI}-30 \mathrm{~mol} \% \mathrm{Al}_{2} \mathrm{O}_{3}$ has a hexagonal structure with $a=4.595 \AA$ and $c=26 \cdot 25 \AA$, where $\mathrm{Ag}$ ions occupy slightly distorted tetrahedral sites. As $\gamma-\mathrm{Al}_{2} \mathrm{O}_{3}$ content is increased to $>60 \mathrm{~mol} \%$, more and more random stacking faults form (as indicated by substantially broadened Bragg reflections) which limit the size of well-ordered $7 \mathrm{H}$ phase domains. Broad transitions in conductivity with large hysteresis characterize a "martensitic transformation' between $\alpha$-AgI and $7 H$-AgI. The $7 \mathrm{H}$ structure is conceived as a heterolayer of $\beta$-AgI and $\gamma$-AgI. It is completely charged, as its thickness would be comparable to or even smaller than the Debye length. Questions for the future are (a) a theory of martensitic phase transformation and (b) long-term stability of the $7 \mathrm{H}$ phase in the composite.

Most of the crystalline superionic conductors are usually synthesized in polycrystalline form either for physical measurements or for applications. These polycrystalline samples contain besides the point defects, substantial concentrations of line defects such as dislocations, planar defects such as stacking faults, free surfaces and grain boundaries, metastable phases synthesized by interfacial phase transitions and mixture of two or more polymorphic phases. Most of these defects enhance ionic conductivity relative to the single crystal phase so that a theoretical model is required to separate the contribution of one or more of these additional contributors, which may often have different and distinct morphologies.

Treating the polycrystalline samples as a composite, an effective medium theory (Bhattacharya et al 1997) has been utilized to express the effective conductivity of the composite in terms of conductivities of phases and their volume fractions. From a knowledge of the effective conductivity $\left(\sigma^{*}\right)$, the conductivity of the other phase $\left(\sigma_{1}\right)$, the volume fraction of the two phases, the conductivity of the unknown phase $\left(\sigma_{2}\right)$ (grain boundary or minor phase or a new polymorph) can be determined by solving the equation,

$$
\begin{aligned}
& \left(\sigma_{1}+2 \sigma *\right) \sigma_{2}^{2}+\left(4 \sigma * \sigma_{1}+8\left(\sigma^{*}\right)^{2}+\right. \\
& \left.9 C \sigma^{*} \sigma_{1}-9 C(\sigma *)^{2}\right) \sigma_{2}-5\left(\sigma^{*}\right)^{2} \sigma_{1}-10\left(\sigma^{*}\right)^{2}+ \\
& 9 C \sigma_{1}\left(\sigma^{*}\right)^{2}-9 C\left(\sigma^{*}\right)^{3}=0,
\end{aligned}
$$

where $C=N_{\mathrm{s}} r_{\mathrm{s}}^{2} /\left(1.5 N_{\mathrm{p}} r_{\mathrm{p}}^{2}\right)$ and $r_{\mathrm{p}}, r_{\mathrm{s}}$ are the radii of the pipe and spheres assumed to make up the composite. $N_{\mathrm{s}} / N_{\mathrm{p}}$ could be evaluated from the dislocation density ' $n$ ' i.e.

$$
N_{\mathrm{p}} / N_{\mathrm{s}}=4 \pi r_{\mathrm{s}}^{2} n \text {. }
$$

This theory has been tested by applying it to $\mathrm{TlCl}, \mathrm{CuCl}$ and $\mathrm{CaF}_{2}$.

\section{Mixed conductors}

Mixed conductors have both mobile ions and conduction electrons which contribute to the total electric current and are important components of solid state battery systems. It is an important model of the conductivity of such systems. Ogawa and Kobayashi (2002) have calculated the conductivity of silver chalcogenides (which involves $\mathrm{Ag}^{+}$ and electrons) by a continuum (hydrodynamic) model considering the force of the electron wind on a mobile ion and vice versa. This force is given by

$$
\mathbf{F}=\hbar \mathbf{k}_{\mathrm{F}} S \mathbf{j}_{\mathrm{e}} / \mathbf{e},
$$

where $\hbar \mathbf{k}_{\mathrm{F}}$ is the Fermi momentum, $\mathbf{j}_{\mathrm{e}}$ the electronic current density and $S$ the electron-atom scattering crosssection.

In this model the superionic conductor is considered as a lattice-fluid system-the lattice of anions immersed in the cation liquid. They show that the ratio of the ionic conductivity to the cross conductivity is given by the ratio of the diffusing particles. Furthermore they show the motion of mobile ions is more properly treated in the spirit of a hopping model with an overlapping of potentials affecting the electrons. This model needs to be applied to a variety of compounds including $\mathrm{AgI}, \mathrm{CuI}$, $\mathrm{Ag}_{3} \mathrm{SI}$ and extended to practical electrolytes and cathodes.

\section{Superionic glasses}

Glasses in general (Rao 2002) and superionic glasses in particular represent systems where short/intermediate range structural order creates very favourable (shallow) potentials for the ionic charges (e.g. $\mathrm{Ag}^{+}$in $\mathrm{AgI}-\mathrm{Ag}_{2} \mathrm{O}-\mathrm{B}_{2} \mathrm{O}_{3}$ glass) to carry out fast hopping motion resulting in enhanced conductivity with respect to one of the constituents. As there is no long range ordered lattice, there is no phase transition characteristic of the crystalline phase (AgI) but only a glass transition for the overall system. Ionic conductivity in glasses is intimately connected to their formation and stabilization. A simple picture of how ions can conduct in a glass is as follows: Initially, in the molten state (prior to quenching) every ion in glass has translational freedom. This is also when each ion feels 
the interionic potentials from all others, which present a frictional force to ion migration and d.c. conductivity. As the temperature is lowered, a given ion suffers a strong interionic force mainly from its neighbours, which form a cage around the given ion. The earlier free translation has now given place to a local, restricted back-and-forth vibrational motion observable in the far infrared region. D.c. conductivity is now possible only if the ion can escape from the cage, creating a free volume in the process or overcoming various potential barriers or finding a percolation path. When the motion of majority of ions are thus restricted by the cage, the system freezes to a glassy state with various local structures frozen in, resulting in a local homogeneity and inhomogeneous distribution. Even in such a situation, ions such as $\mathrm{Li}^{+}$and $\mathrm{Ag}^{+}$can find escape routes in view of their small size, large polarizability or the existence of diffusion paths by clusters (such as AgI).

Burt has described a $60 \mathrm{~W}$-bulb experiment (Sunandana 1995), which dramatically demonstrates d.c. ionic conduction in a glass.

Frequency dependent conductivity of fast ion conducting glasses can be analysed using the phenomenological formula of Almond and West (Hairetdinov et al 1994).

$$
\sigma(v)=\sigma_{\text {d.c. }}\left[1+\left(v / v_{H}\right)^{\alpha}\right],
$$

where $\sigma_{\text {d.c. }}$ is low frequency limit of bulk conductivity, $v$ the applied electrostatic frequency, $v_{\mathrm{H}}$ the hopping frequency of the charge carriers and $\alpha$ is a dimensionless parameter, $0 \leq \alpha \leq 1$.

D.c. conductivity $\left(\sigma_{\text {d.c. }}\right)$ arises from long range ion motion. For a single ion conductor (e.g. $\mathrm{Ag}^{+}$in $\mathrm{AgCl}$ ),

$$
\sigma_{\text {d.c. }}=(Z e) n \mu,
$$

where the concentration of the mobile ions ' $n$ ' is given by

$$
\begin{aligned}
n & =N_{0} \exp \left(-\Delta G_{\mathrm{e}} / k T\right) \\
& =N_{0} \exp \left(\Delta S_{\mathrm{c}} / k T\right) \exp \left(-\Delta H_{\mathrm{c}} / k T\right) \\
& =N_{\mathrm{e}} \exp \left(-\Delta \mathrm{H}_{\mathrm{c}} / k T\right),
\end{aligned}
$$

where $\Delta G_{\mathrm{e}}$ is the free energy necessary to raise the carrier population, $\Delta S_{\mathrm{c}}$ the entropy, $\Delta H$ the enthalpy and $N_{\mathrm{e}}$ the effective intermediate temperature ion concentration (including entropy).

Mobility ' $\mu$ ' is related to diffusion $(D)$ through Nernst Einstein relation,

$$
\begin{aligned}
\mu & =(Z e / k T) D=(Z e / k T) \chi \lambda^{2} v_{\mathrm{H}} \\
& =(Z e / k T) \gamma \lambda^{2} v_{0} \exp \left(-\Delta G_{\mathrm{m}} / k T\right) \\
& =(Z e / k T) \gamma \lambda^{2} v_{0} \exp \left(\Delta S_{\mathrm{m}} / k T\right) \exp \left(-\Delta H_{\mathrm{m}} / k T\right) \\
& =(Z e / k T) \gamma \lambda^{2} v_{\mathrm{e}} \exp \left(-\Delta H_{\mathrm{m}} / k T\right),
\end{aligned}
$$

where $\Delta G_{\mathrm{m}}$ is the free energy for ion migration, $\Delta S_{\mathrm{m}}$ the entropy, $\Delta H_{\mathrm{m}}$ the enthalpy, $\gamma$ the geometric factor for ion hopping, $\lambda$ the average jump distance between mobile ion sites, $v_{\mathrm{H}}$ the actual jump frequency, $v_{0}$ the jump attempt frequency of mobile ions ( $\sim$ mobile ion resonance fre- quency) and $v_{\mathrm{e}}$ the effective jump attempt frequency including entropy term.

$$
\mathrm{v}_{\mathrm{H}}=\mathrm{v}_{\mathrm{e}} \exp \left(-\Delta E_{\mathrm{m}} / k T\right) .
$$

(As $\Delta H_{\mathrm{m}}=\Delta E_{\mathrm{m}}$ from first law of thermodynamics, under conditions of constant sample volume and temperature). Thus,

$$
\begin{aligned}
& \sigma_{\text {d.c. }}(\text { theory })=\left(N_{\mathrm{e}}(Z e)^{2} / k T\right) \gamma \lambda^{2} v_{\mathrm{e}} \\
& \exp \left\{-\left(\Delta E_{\mathrm{c}}+\Delta E_{\mathrm{m}}\right) / k T\right\}, \\
& \sigma_{\text {d.c. }}(\operatorname{expt} .)=\sigma_{0} \exp \left(-\Delta E_{\mathrm{a}} / k T\right),
\end{aligned}
$$

where $\sigma_{0}$ is the infinite temperature limit of d.c. conductivity and $\Delta E_{\mathrm{a}}$ the measured activation energy for d.c. conduction.

We cannot separate $\Delta E_{\mathrm{c}}$ and $\Delta E_{\mathrm{m}}$ from measured $\Delta E_{\mathrm{a}}$. Furthermore, pre-exponent $\sigma_{0}$ contains several individual parameters, $\gamma, \lambda, N_{\mathrm{e}}$ and $v_{\mathrm{e}}$, which are again inseparable. Thus, $\sigma_{\text {d.c. }}$ cannot be used to infer the ionic conduction mechanism in glasses unless independent tracer diffusion measurements are carried out.

Frequency dependent conductivity $\left(\sigma_{\text {a.c. }}\right)$ enables $\Delta E_{\mathrm{c}}$ and $\Delta E_{\mathrm{m}}$ to be separated from the measured activation energy. The power law equation of Almond and West empirically describes the conductivity dispersion.

$$
\sigma^{\prime}(\omega)=\sigma_{\text {d.c. }}+A \omega^{\alpha} \text {. }
$$

Dielectric loss is related to real part of complex conductivity through

$$
\sigma^{\prime}(\omega)=\omega \chi^{\prime \prime}(\omega)
$$

where

$$
\chi^{\prime \prime}(\omega) \propto\left[\omega / \omega_{\mathrm{p}}\right]^{m}+\left[\omega / \omega_{\mathrm{p}}\right]^{n-1} .
$$

$\omega_{\mathrm{p}}=2 \pi v_{\mathrm{p}}-$ dielectric loss peak frequency, ' $m$ ' and ' $n$ ' are parameters describing high and low frequency slopes, respectively of dielectric loss peak ( $m \sim 1$ and $n \sim 0.5)$.

$$
\therefore \sigma^{\prime}(\omega) \propto \omega\left\{\left[\omega / \omega_{\mathrm{p}}\right]^{m}+\left[\omega / \omega_{\mathrm{p}}\right]^{n-1}\right\} .
$$

Let $k$ be the common proportionality constant in $\chi^{\prime \prime}(\omega)$ and $\sigma^{\prime}(\omega)$. Also, let $\omega_{\mathrm{p}}=\omega_{\mathrm{H}}=2 \pi \nu_{\mathrm{H}}$ and $m=-1, n=\alpha$,

$$
\begin{aligned}
\sigma^{\prime}(\omega) & =k a\left\{\left[\omega / \omega_{\mathrm{H}}\right]^{-1}+\left[\omega / \omega_{\mathrm{H}}\right]^{\alpha-1}\right\} \\
& =k \omega_{\mathrm{H}}+k \omega_{\mathrm{H}}{ }^{1-\alpha} \omega^{\alpha},
\end{aligned}
$$

comparing to $\sigma^{\prime}(\omega)=\sigma_{\text {d.c. }}+A \omega^{\alpha}$

$$
\sigma_{\text {d.c. }}=k \omega_{\mathrm{H}} \text {. }
$$

$k$ is found to be composed of carrier concentration, geometric factor, coulomb energy and Boltzmann constant. A.c. and d.c. conductivity terms of $\sigma^{\prime}(\omega)$ are related to each other in terms of $A$, i.e.

$$
\begin{aligned}
& \sigma_{\text {d.c. }}=A \omega_{\mathrm{H}}^{\alpha}, \\
& \sigma^{\prime}(\omega)=\sigma_{\text {d.c. }}+\sigma_{\text {d.c. }}\left(\omega / \omega_{\mathrm{p}}\right)^{\alpha}=\sigma_{\text {d.c. }}\left[1+\left(\omega / \omega_{\mathrm{p}}\right)^{\alpha}\right],
\end{aligned}
$$




$$
\begin{aligned}
& \sigma^{\prime}(v)=\sigma_{\text {d.c. }}\left[1+\left(v / v_{p}\right)^{\alpha}\right], \\
& \sigma^{\prime}\left(v_{H}\right)=2 \sigma_{\text {d.c. }} .
\end{aligned}
$$

Thus, $v_{H}$ can be obtained from the analysis of a.c. conductivity. Bulk a.c. conductivity in terms of physical parameters is therefore,

$$
\begin{aligned}
\sigma^{\prime}(v)= & \left(N_{\mathrm{e}}(Z e)^{2} / k T\right) \gamma \lambda^{2} v_{\mathrm{e}} \exp \left\{-\left(\Delta E_{\mathrm{c}}+\Delta E_{\mathrm{m}}\right) / k T\right\} \\
& {\left[1+\left(\mathrm{v} / v_{\mathrm{e}} \exp \left(-\Delta E_{\mathrm{m}} / k T\right)\right)^{\alpha}\right] . }
\end{aligned}
$$

From (51), activation energies for $\mathrm{v}_{\mathrm{H}}, \sigma_{\mathrm{d} . c .}, A$ are $\Delta E_{\mathrm{c}}$, $\Delta E_{\mathrm{c}}+\Delta E_{\mathrm{m}}$ and $\Delta E_{\mathrm{c}}+(1-\alpha) \Delta E_{\mathrm{m}}$, respectively. If the first two activation energies are the same, then $\Delta E_{\mathrm{c}} \approx 0$ and charge carrier concentration is temperature independent. All ions are essentially mobile and support the strong electrolyte model for glasses. If they are different, then the carrier concentration is thermally activated and supports the weak electrolyte model for these glasses. This formalism has been used to analyse the frequency dependent conductivity of $\mathrm{Na}_{2} \mathrm{~S}-(1-x) \quad \mathrm{B}_{2} \mathrm{~S}_{3} \quad(0 \cdot 001 \leq x$ $\leq 0 \cdot 1)$ glasses.
Mobile ion hopping dynamics (at the microscopic state) in these and other glasses is fundamental, challenging and crucial to the understanding of the ion conduction process. Realize that the 'attempt frequency' of the frequency dependent conductivity with temperature as parameter studied in the widest possible range from subacoustic to microwave frequencies has provided a very good basis for the testing of various theoretical models for conduction. Figure 6 shows the typical conductivity spectra for three glass systems: $0.45 \mathrm{LiBr}-0.56 \mathrm{Li}_{2} \mathrm{O}-\mathrm{B}_{2} \mathrm{O}_{3}$ (Funke 1997), $\mathrm{Na}_{2} \mathrm{O}-3 \mathrm{SiO}_{2}$ (Jain 1999). As many as eight regimes are identified with $\omega$ in the range $(\mathrm{Hz}-$ far IR) and $T$ in the range $T_{\mathrm{g}}<T<$ a few $K$ (table 1 ).

Three remarkable features characterize the conductivity of glasses.

(I) Unlike in crystalline systems where an ion can hop to a neighbouring site only if the latter exists, in a glass, mobile ions are by themselves able to create vacant sites with suitable conformations, paving the way for ion transport. This is the essence of the so-called dynamical
(A)

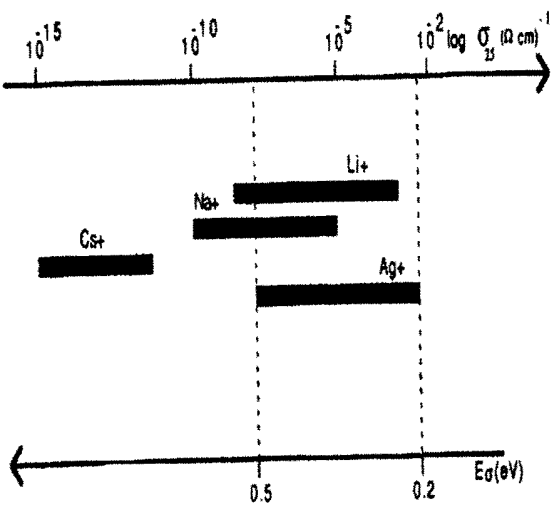

(C)

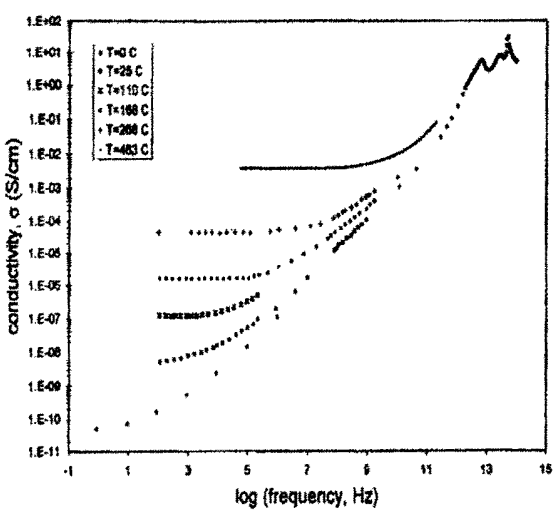

(B)

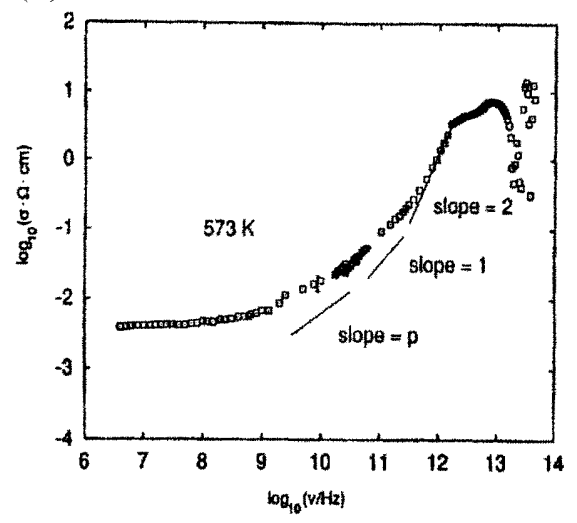

(D)

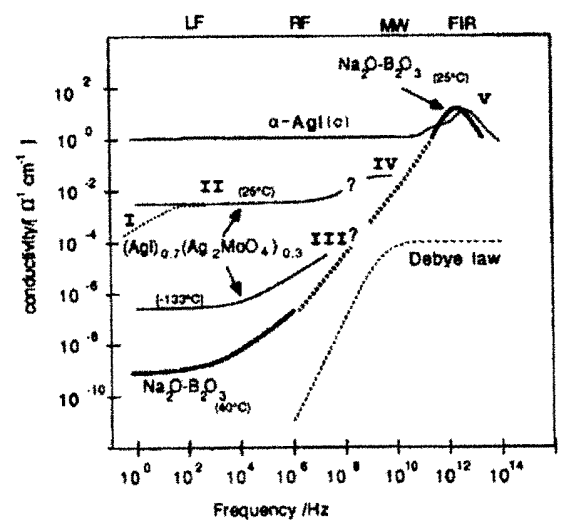

Figure 6. (A) Ranges of $\mathrm{Li}^{+}, \mathrm{Na}^{+}, \mathrm{Ag}^{+}$and $\mathrm{Cs}^{+}$ion conductivity and their activation energies in glasses, (B) frequency dependent conductivity in $0.45 \mathrm{LiBr}-0 \cdot 56 \mathrm{Li}_{2} \mathrm{O}-\mathrm{B}_{2} \mathrm{O}_{3}$ at $573 \mathrm{~K}$; (C) $\mathrm{Na}_{2} \mathrm{O}-3 \mathrm{SiO}_{2}$ glass at various temperatures in the range $0-483^{\circ} \mathrm{C}$; (D) $(\mathrm{AgI})_{0.7}$ $\left(\mathrm{Ag}_{2} \mathrm{MoO}_{4}\right)_{0.3}$ glass at $25^{\circ} \mathrm{C}$ and $-133^{\circ} \mathrm{C}$, as compared with crystalline $\alpha-\mathrm{AgI}$ and $\mathrm{Na}_{2} \mathrm{O}-\mathrm{B}_{2} \mathrm{O}_{3}$. 
structural model (Funke et al 1996, 1997; Ingram 1998) still in the making (figure 7a).

(II) The large conductivity of an AgI-based glass relative to the crystal of the same composition suggests that the glass must contain a large number of mobile ions (repelling each other) and an even larger number of sites (available for hopping) than the number of mobile ions. (Such a situation occurs in $\alpha$-AgI where 48 sites are available for the hopping of just $2 \mathrm{Ag}^{+}$ions in the $b c c$ unit cell of $\alpha-\mathrm{AgI})$. Thus arises the basic idea of the jump relaxation model as in figure $7 \mathrm{~b}$. The presence of the cage around a given hopping ion ensures that there is a finite distance between such ions. If at $t=0$, an ion hops to a neighbouring site, then a mismatch occurs. After the hop, (a) the ion may hop back to its original site or (b) the neighbouring mobile ions rearrange during their own hopping motion. Situation (b) results in a shifting of the cage effect potential. A new 'global' minimum is formed for the given ion at its new site. The initial forward hop has succeeded and d.c. conduction occurs. In this model, the frequency dependent conductivity is given as

$$
\sigma(\omega)=\sigma(\infty)\left[1+\left(1 / \omega t_{1}\right)\right]^{-s} \text { at } \omega \gg t_{2}{ }^{-1} .
$$

Here, $\sigma(\infty)$ is the high frequency conductivity, $t_{1}$ and $t_{2}$ are the time at which the conductivity dispersion starts and ends, respectively and $s$ the Jonscher power law exponent $\left(\sigma(\omega) \propto \omega^{s}\right)$. The experimental data, however, fit the expression

$$
\sigma_{\text {hop }}(\omega)-\sigma(0)=A\left[1+\left(1 / \omega t_{1}\right)\right]^{-s}+B\left[1+\left(1 / \omega t_{1}\right)\right]^{-s^{\prime}},
$$

where $s$ and $s^{\prime}$ are different. For the $0.45 \mathrm{LiBr}-0.56$ $\mathrm{Li}_{2} \mathrm{O}-\mathrm{B}_{2} \mathrm{O}_{3}$ glass, $s=0.6$ and $s^{\prime}=1 \cdot 3$. The appearance of the second exponential calls for an explanation. This has been provided by including the essential feature of the dynamic structure model in the jump relaxation model. (III) The low temperature $(<200 \mathrm{~K})$ low frequency $(<\mathrm{MHz})$ conductivity behaviour of glasses (e.g. $0 \cdot 2 \mathrm{~K}_{2} \mathrm{O}-$ $0.98 \mathrm{GeO}_{2}$ ) with moderately high conductivity has thrown up unusual phenomena, quite distinct from $\sigma_{\text {d.c. }}$ or a universal dynamic response.

Power laws in both $T$ and $\omega$ are observed.

$$
\sigma(\omega, T) \propto T^{\alpha} \omega^{\beta}(0 \leq \alpha \leq 0 \cdot 25 ; 1 \cdot 0 \leq \beta \leq 1 \cdot 15) .
$$

What kind of ion displacements result in the observed a.c. conductivity in this region? An asymmetric double well potential (ADWP) or a 'jellyfish' atom model (Jain 1999) has been invoked to describe these observations. 'Jellyfish' refers to a group of atoms whose 'wiggling' motion requires small energy of $\sim 50 \mathrm{meV}$. A change in the conformation of atoms, considered as an excitation over an asymmetric energy barrier from one potential well to another, is thought to give rise to the observed a.c. conductivity. In this model,

$$
\begin{gathered}
\sigma(\omega, T)=\left(\omega N e^{2} R^{2} / 12 k T\right) \int_{0}^{V_{\mathrm{m}}} \int_{0}^{\Delta_{\mathrm{m}}} \sec \hbar^{2}(\Delta / 2 k T) \\
f(\Delta, V)\left(\omega \tau /\left(1+(\omega \tau)^{2}\right) \mathrm{d} \Delta \mathrm{d} V,\right.
\end{gathered}
$$

where $N$ is the ion concentration, $V$ the average potential barrier, $\Delta$ the asymmetry energy, $\tau$ the relaxation time, $R$ the inter-atomic separation, $f(\Delta, V)$ the distribution of ADWP conformations, with $V_{\mathrm{m}}$ and $\Delta_{\mathrm{m}}$ as maximum values, $k, T$ and $e$ have usual meanings. At sufficiently low temperatures, this equation simplifies to the observed power law, $\sigma(\omega, T) \propto T^{\alpha} \omega^{\beta}$. This conductivity regime is

\begin{tabular}{|c|c|c|c|}
\hline & $\begin{array}{l}\text { Typical } T \\
\text { range }\end{array}$ & Typical $\omega$ range & Typical characteristics \\
\hline Region (a) & $\begin{array}{l}\text { Approx. } \\
R T \leq T \leq T_{\mathrm{g}}\end{array}$ & Low, $\mathrm{Hz}-\mathrm{kHz}$ & $\begin{array}{l}\sigma \text { is } \omega \text { independent, thermally activated with energy } E_{\text {d.c. }} \Rightarrow \text { d.c. conductivity, } \\
\sigma_{\text {d.c. }} \text { from ion hopping }\end{array}$ \\
\hline Region (b) & $\begin{array}{l}\text { Approx. } \\
R T \leq T \leq T_{\mathrm{g}}\end{array}$ & $\begin{array}{l}\text { Medium high, up } \\
\text { to several } \mathrm{MHz}\end{array}$ & $\begin{array}{l}\text { Onset of a.c. dispersion, } \sigma_{\text {a.c. }}=A \omega^{s}, s \sim 0 \cdot 5-0 \cdot 65 \text {. Non-Debye behaviour. } \\
A \text { is thermally activated and } E_{\text {a.c. }} \approx s E_{\text {d.c. }}\end{array}$ \\
\hline Region (c) & $\begin{array}{l}\text { Approx. } \\
R T \leq T \leq T_{\mathrm{g}}\end{array}$ & $\mathrm{MHz}-\mathrm{GHz}$ & $\begin{array}{l}\sigma_{\mathrm{HTHF}} \sim A^{\prime} \omega^{s^{\prime}}, s^{\prime} \approx 1 \text {. Too few data to define weak } T \text { dependence. FICs do not show } \\
\text { this behaviour, rather region (b) persists }\end{array}$ \\
\hline Region (d) & $\begin{array}{l}\text { Approx. } \\
R T \leq T \leq T_{\mathrm{g}}\end{array}$ & $\begin{array}{l}\mathrm{GHz} \text { to below } \\
\text { far-IR }\end{array}$ & $\begin{array}{l}\text { Shown by FIC crystals and glasses, as the second plateau i.e. } \sigma \text { is again } \omega \text { inde- } \\
\text { pendent and thermally activated }\end{array}$ \\
\hline Region (e) & $\begin{array}{l}\text { Approx. } \\
R T \leq T \leq T_{\mathrm{g}}\end{array}$ & Far-IR & $\begin{array}{l}\omega \text { exponent } s^{\prime} \text { approaches } 2 \text {, followed by a peak in } \sigma \text { due to local ion vibrations, } \\
\text { which is practically } T \text { independent }\end{array}$ \\
\hline Region (f) & $\begin{array}{l}\text { Approx. } \\
200 K \geq T \geq \\
\text { few } K\end{array}$ & $\begin{array}{l}\text { Low to medium; } \\
\leq \mathrm{MHz}\end{array}$ & $\begin{array}{l}\text { For glasses with moderate to high } \sigma_{\text {d.c.; }} ; \sigma(\omega, T) \sim T^{\alpha} \omega^{\beta} \text { where } 0 \cdot 25 \geq \alpha \geq 0 \text { and } \\
1 \cdot 15 \geq \beta \geq 1 \cdot 0 . \text { For low } \sigma_{\text {d.c. }} \text { glass: } T \text { and } \omega \text { dependence is complex showing peaks }\end{array}$ \\
\hline Region (g) & $\begin{array}{l}\text { Approx. } \\
200 K \geq T \geq \\
\text { few } K\end{array}$ & $>\mathrm{MHz}$ & Little information to identify overall characteristics \\
\hline Region (h) & $T<$ few $\mathrm{K}$ & Low to high & Complex $T$ and $\omega$ dependence from tunneling within two-level systems (TLS) \\
\hline
\end{tabular}
one of nearly constant loss and has been called a new universality of ion dynamics.

Table 1. Separation of electrical conductivity of ionic glasses into various regions of $\omega$ and $T$, based on empirical observations (after Jain 1999). 
An experimentally observed increase in the d.c. conductivity of glasses doped with halides can be explained in terms of an increase in free volume. The conductivity varies as the third power of relative increase in volume. This free volume of excluded volume is also held responsible for superionic conductivity in $\mathrm{CuI}$ and possibly in $\mathrm{CuI}$ based solid solutions with $\mathrm{AgI}$ as well as in $\mathrm{KCu}_{4} \mathrm{I}_{5}$.

The d.c. conductivity of glassy fast ion conductors does not follow a simple Arrhenius type law (Kawamura and Shimoji 1986; Maass et al 1996) below the glass transition temperature i.e. the relation,

$$
\sigma_{\text {d.c. }} T=A_{0} \exp \left(-E_{\mathrm{a}} / k T\right),
$$

is violated. At low temperatures, such a relation is obeyed, where $E_{\mathrm{a}}$ is the activation energy due to the effects of energetic disorder and interaction. At high temperatures, 'bare interaction' dominates and limits conductivity leading to non-Arrhenius behaviour.

In the 'mode coupling phenomenological theory' of Ngai (1980), the non-Arrhenius behaviour is explained as follows: within the mode coupling concept, the temperature dependent activation energy, $E_{\mathrm{a}}(T)$, is interpreted as a crossover from a high value at low $T$, where the conductivity relaxation is dominated by a Kohlrausch-WilliamWatts behaviour, to a smaller value of $E_{\mathrm{a}}$ at high $T$, where it is dominated by an exponential relaxation function.

Ishii and Abe (1999) proposed an intrinsic mechanism of the universality having the double power law ( $s$ and $s^{\prime}$ in (52)) in a unified manner in terms of the diffusion length of particles. The non-Debye relaxation represented by

$$
\sigma^{\prime}(\omega)=\sigma(0)+A \omega^{s}+A^{\prime} \omega^{s \prime}\left(s \approx 0 \cdot 6, s^{\prime} \approx 1\right),
$$

originates from a power law distribution of the mode diffusion lengths. Here, the second term is important at low frequencies and high temperatures while the third term dominates at high frequencies and low temperatures.
A relaxation mode theory defines the probability of finding a particle of size ' $n$ ' at time ' $t$ ' i.e. $p_{n}(t)$. The time evolution of $p_{n}(t)$ is governed by the master equation. The eigenvalue equation,

$$
H\left|\psi_{\mathrm{q}, \varepsilon}\right\rangle=E_{\mathrm{q}, \varepsilon}\left|\psi_{\mathrm{q}, \varepsilon}\right\rangle .
$$

A hopping operator, $H$, is defined:

$$
H_{\mathrm{nm}}=\Sigma W_{n^{\prime} m} n=m, \sqrt{ } W_{n m} W_{m n} n \neq m .
$$

$W_{n m}$ being the transition rate, $W_{n m}$, from site ' $m$ ' to site ' $n$ '. ' $W$ ' could correspond to a supercell (spacing ' $a$ ') as in molecular dynamics simulations with two energy barriers, $\Gamma$ and $(l-1) \gamma$, where $l$ is the number of ions, or a double well lattice (spacing ' $a$ ') with the same kind of energy barriers. These give one diffusing relaxation mode and $(l-1)$ non-diffusive modes. $E_{\mathrm{q}, \varepsilon}$ is the eigenvalue of the relaxation mode at wavenumber ' $q$ ' for branch ' $\varepsilon$ ' and $\left|\psi_{\mathrm{q}, \varepsilon}\right\rangle$ is the eigenfunction defined by $\psi=p / \phi$ with $\phi^{2}=p_{\text {eq }}, p_{\text {eq }}$ being the equilibrium probability.

The diffusive mode, $\varepsilon=0$, and extended non-diffusive modes, $1 \leq \varepsilon \leq l-2$, contribute to d.c. conductivity and a.c. conductivity, respectively. The localized mode, $\varepsilon=l-1$, has the largest eigenvalue and contributes to a.c. conductivity. Using the diffusion length and density of states for relaxation modes, real conductivity is approximately,

$$
\sigma_{\text {extended }}^{\prime}(\omega)=\operatorname{Re}\left\{\sigma_{\text {extended }}(\omega)\right\} \text {. }
$$

Furthermore,

$$
s=2-\alpha-\delta,
$$

where $\alpha$ characterizes the diffusion length distribution exponent and $\delta$ is the density of states exponent (of relaxation mode).

$$
A=K(\pi / 2) \beta n_{0}(1-\Gamma)(e a)^{2} r^{1-s} \operatorname{cosec}(s \pi / L) .
$$

$K=$ constant, density of states parameter $B$ is given by
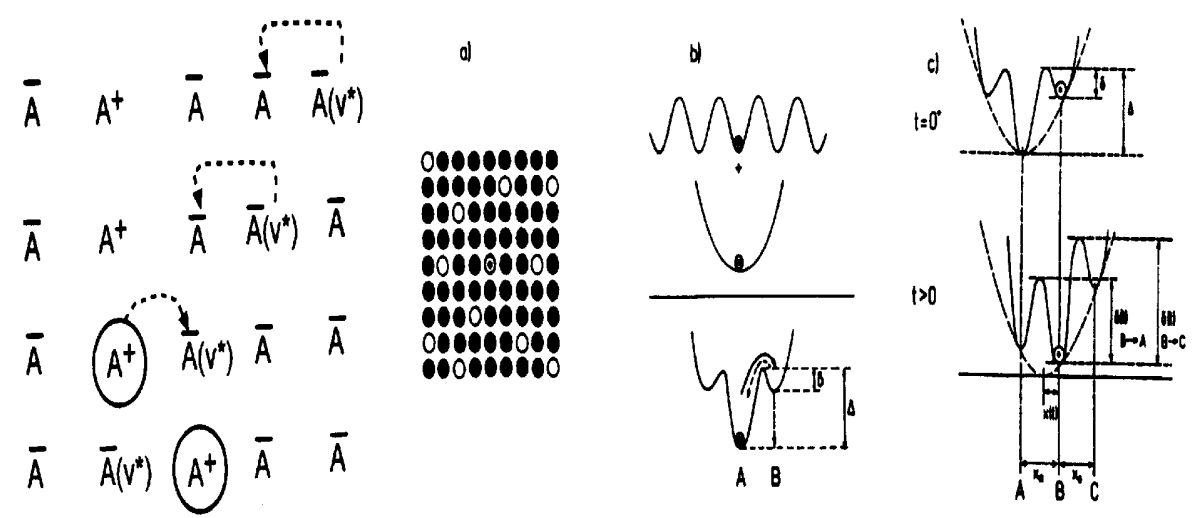

Figure 7. Two models for ion transport in glasses. Left: Dynamic structure model in which ion hopping is assisted by free volume migration within a 'rigid' glass and right: Jump relaxation model: (a) ions in a sublattice, (b) effective single ion potential and (c) development of the potential after an ion hop (Funke 1996, 1997; Ingram 1998). 
$D\left(E_{0, \varepsilon}\right)=B r^{-1}\left(E_{0, \varepsilon} / r\right)^{-f}$ in the frequency range $E_{0,1} \leq \omega \leq$ $E_{0,1-2}, n_{0}$ the mobile ion density, $e$ the electric charge and $a$ the nearest neighbour spacing of the lattice.

A major result of this model is

$$
A \approx \exp \left(-\beta(1-s) U_{\mathrm{r}}\right),
$$

where $\beta=1 / k_{\mathrm{B}} T$ and $U_{\mathrm{r}}$ is the intrinsic activation energy. This represents the non-Debye behaviour.

The mode diffusion length, $l_{\varepsilon, \mathrm{q}}=|\phi| R\left|\psi_{\mathrm{q}, \varepsilon}\right|$, where $R$ is the site position, decreases with increasing relaxation eigenvalue and is expected to asymptotically saturate to a constant value since $\psi_{0, \varepsilon}$ tends to localize for large eigenvalues. Thus arises another exponent for localized conductivity, $\sigma_{\text {loc }}(\omega)$. Therefore, for particle localization,

$$
\begin{aligned}
& \sigma_{\text {loc }}^{\prime}(\omega)=\operatorname{Re}\left\{\sigma_{\text {loc }}(\omega)\right\}=A^{\prime} \omega^{s \prime}, \\
& s^{\prime}=\eta-\alpha-\delta ; \alpha=0 .
\end{aligned}
$$

Finally,

$$
\sigma_{\mathrm{loc}}^{\prime}(\omega) \approx \omega^{2} \beta U\left(\eta /\left(\eta^{2}+\omega^{2}\right)\right),
$$

where $\eta=\omega_{\mathrm{s}} \exp (-\beta U), \omega_{\mathrm{s}}$ being attempt frequency and $A^{\prime}$ being a constant independent of temperature.

Experimental conductivity spectra, $\sigma(\omega)$, of number of superionics have been formally described in the concept of mismatch and relaxation (Funke and Wilmer 2000). An experimental master curve is obtained by superimposing and shifting conductivity spectra along the $\sigma$ and $\omega$ axes simultaneously in the spirit of the time-temperature superposition principle. The relation (for frequencies below $10 \mathrm{MHz}$ i.e. low frequency),

$$
\ln [\sigma(\omega) / \sigma(0)]=E_{1}^{-1}\left\{\omega_{\text {onset }} / \omega\right\},
$$

where $E_{1}^{-1}$ is the inverse of the exponential integral, reproduces the experimental data. Here, $\sigma(\omega) / \sigma(0)$ has a fixed numerical value of 1.303 at $\omega=\omega_{\text {onset }}$. Note that it contains only $\sigma(0)$ and $\omega_{\text {onset }}$ required for normalization. No adjustable parameter is involved. At high frequencies (mm wave range), $\sigma(\omega) \rightarrow \sigma(\infty)$. Incorporating this into the above equation, we could independently write,

$$
t / t_{\text {onset }}=E_{1}\left(\ln (D(t) / D(\infty))-E_{1}(\ln (D(0) / D(\infty))),\right.
$$

where

$$
t_{\text {onset }}=\left(\omega_{\text {onset }} \cdot \pi / 2\right)^{-1},
$$

neglecting cross terms in velocity autocorrelation, apply Nernst-Einstein relation, the $t / t_{\text {onset }}$ relation yields $\omega_{\text {onset }} / \omega$ by the Fourier transformation of $\mathrm{d} D(t) / \mathrm{d} t$.

At any temperature, the typical elementary hopping distance is given by the relation

$$
X_{0}^{2}=6 D(\infty) t_{\text {onset }}
$$

Let us define two functions, $f(t)$ and $g(t)$.

$$
f(t)=\ln (D(t) / D(\infty)) ; \quad g(t)=\exp \left(-\left\langle r^{2}(t)\right\rangle / x_{0}^{2}\right) .
$$

Note that $D(t)$ is related to $\left\langle r^{2}(t)\right\rangle$ and $D(\infty)$ is related to $x_{0}^{2}$ so that $f(t)$ and $g(t)$ are both 'entangled'. $f(t)$ and $g(t)$ are related by

$$
f(t)=\ln (D(t) / D(\infty)) g(t) .
$$

The self-neighbourhood created by the mismatch ion when it jumps to a neighbouring site is now interpreted as the distance between the ion and a position at which it would be optimally relaxed with respect to the momentary arrangement of its mobile neighbours. If the cage-effect potential is harmonic then the mismatch is proportional to the backward driving force exerted by its neighbourhood. The original mismatch is relaxed by the system in course of time. Two possible modes arise: one single-particle mode in which the ion performs a correlated backward hop and a many-particle mode that involves rearrangement of neighbouring ions. The rates for
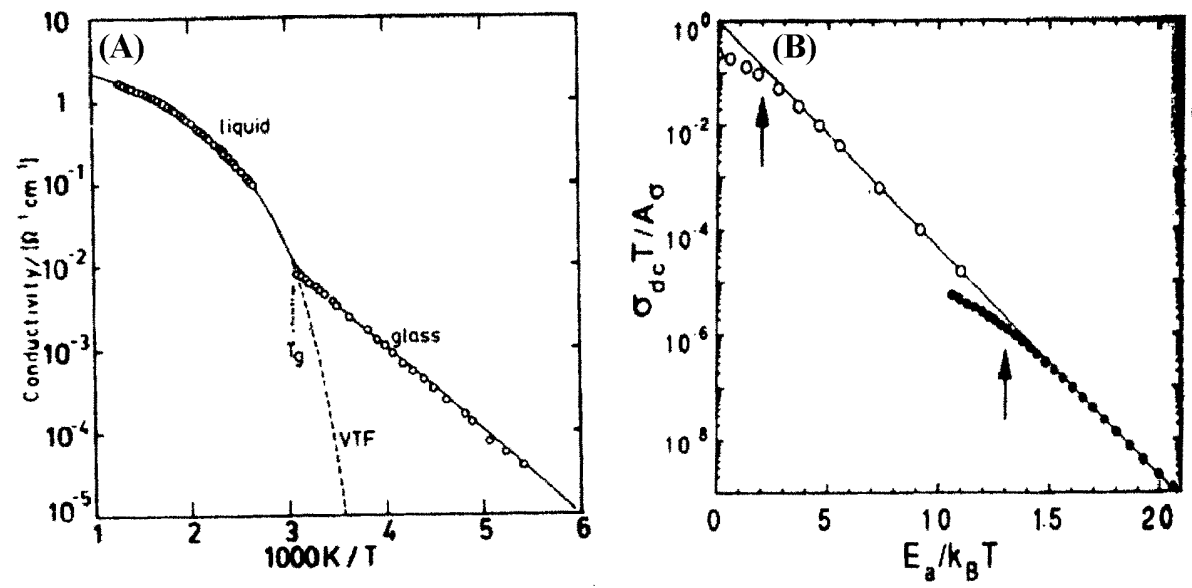

Figure 8. Non-Arrhenius conductivity behaviour below $T_{\mathrm{g}}$. (A) $0 \cdot 7 \mathrm{AgI}-0 \cdot 3 \mathrm{AgMoO}_{4}$ (Kamishima and Shimoji 1986), (B) 0.4AgI-0.6[0.525 $\left.\mathrm{Ag}_{2} \mathrm{~S}+0 \cdot 475\left(\mathrm{~B}_{2} \mathrm{~S}_{3}: \mathrm{SiS}_{2}\right)\right]$ (Hairetdinov 1994). 
the two mismatch relaxation processes are $-\mathrm{d} f(t) / \mathrm{d} t$ and $-\mathrm{d} g(t) / \mathrm{d} t$. Ln $(D(0) / D(\infty))$ is the proportionality constant that relates these two processes. Backward hop tendency is proportional to the tendency for its neighbourhood to rearrange. Model conductivity spectra may be constructed based on the relation,

$$
-\mathrm{d} f(t) / \mathrm{d} t \propto-\mathrm{d} g(t) / \mathrm{d} t
$$

This approach helps to integrate structural features like inhomogeneities of glassy electrolytes and explain the more gradual onset of dispersion and $\omega_{\text {onset }}$ in conductivity spectra.

Govindaraj and Murugaraj (2000) have sought to model the conductivity of disordered materials by introducing a complex phase relaxation function

$$
\phi^{*}(t)=\exp [-t / \tau *]
$$

where

$$
\tau^{*}=\tau^{g} / I^{(1-g)}, i=\sqrt{ }-1 .
$$

The exponent $g$ is the phase or loss factor related to the impedance loss through the $\tan \left(\delta_{\mathrm{p}}\right)$ at the impedance loss (of non-Debye type) frequency, $\omega_{\mathrm{p}}=1 / \tau^{g} \cdot g\left(\delta_{\mathrm{p}}\right)$ arises from the absence of periodicity in the potential in a glass. A notable feature of this model is the absence of a frequency exponent. The frequency-dependent conduction process involves hopping of charge carriers in an environment comprising spatially randomly varying free energy barriers. They have applied this model to fit the conductivity data of $\mathrm{Li}_{4} \mathrm{SiO}_{4}$ and obtained reasonable agreement.

Electronegativity is an attractive concept that gives a reasonable description of the ionicity of the chemical bond (Sunandana 1997). Along with valence and atomic size, it helps build a model for the superionic bond (Sunandana and Senthil Kumar 2003). Many physical properties (e.g. band gap, conductivity) are connected to the nature of this model. Glass formation has been discussed earlier.

A deeply intuitive but readily testable chemical model for ion transport in AgI-based fast-ion conducting oxyanion glasses $\left(\mathrm{AgI}-\mathrm{Ag}_{2} \mathrm{O}-\mathrm{X}_{m} \mathrm{O}_{n}\right.$ where $\mathrm{X}_{m} \mathrm{O}_{n}=\mathrm{B}_{2} \mathrm{O}_{3}, \mathrm{As}_{2} \mathrm{O}_{3}$, $\mathrm{CrO}_{3}, \mathrm{WO}_{3}, \mathrm{MoO}_{3}, \mathrm{GeO}_{2}, \mathrm{P}_{2} \mathrm{O}_{5}, \mathrm{~V}_{2} \mathrm{O}_{5}$ ) has been proposed by Shastry and Rao (1989). This structural unpinning number (SUN) model is based on the attractive and reasonable concept of reverse transfer of electron density to $\mathrm{Ag}^{+}$which brings in electronegativities of $\mathrm{I}^{-}$and oxyanion right from the start. It has been convincingly argued that the ionic conductivity of these glasses must be proportional to the extent of reverse charge transfer and to the free volume available per $\mathrm{Ag}^{+}$ion. Most significantly SUN is a descriptor of conductivity defined as

$$
S=C\left(z^{*} / \chi_{\mathrm{av}}\right)\left\{V_{\mathrm{m}} / N\right)
$$

where $C$ is a constant $\left(1.0 \mathrm{~cm}^{-3.5}\right)$ that makes $S$ dimensionless, $z^{*}$ the unscreened charge on $\mathrm{Ag}$ ion, $\chi_{\mathrm{av}}$ the mole-fraction weighted average electronegativity $f \chi_{\mathrm{I}}^{-}+$ $(1-f) \chi_{\text {oxyanion }}, V_{\mathrm{m}}$ the molar volume of the glass and $N$ the mole number of the $\mathrm{Ag}$ ions, respectively. Structural unpinning of the Ag ions in the glass network from their 'official' positions in the cation sublattice is essentially induced by the slower conductivity relaxation relative to structural relaxation.

Writing the conductivity of a given AgI-oxyanion glass series at room temperature or at $0.8 T_{\mathrm{g}}\left(T_{\mathrm{g}}=\right.$ glass transition temperature) as

$$
\ln \sigma=\ln \sigma_{0} /[1-\exp (-a S)],
$$

where $\sigma_{0}$ is the conductivity of a hypothetical AgI glass and $a$ a constant, one can incorporate the crucial fact that $\sigma$ is a smooth and uniformly increasing function of $S$.

The conductivity activation energies show an Arrhenius-like variation with $S$ when plotted on a semilog scale so that one could write

$$
-\ln E_{\mathrm{a}} \cong \ln \sigma_{0}[1+\exp (-a S)],
$$

in the limit of small exponential. From (77) and (78) one gets

$$
-\ln E_{\mathrm{a}}=a S+\left[\ln R T+\ln \ln \left(1 / \sigma_{0}\right)\right] .
$$

A plot of $\ln E_{\text {a }}$ vs $S$ gives a 'family' of curves, for the various AgI-oxyanion glass series, converging at a point defining the upper limit of AgI concentrations beyond which no useful increase of $\sigma$ occurs. These plots glaringly reflect an increase of $S$ due to diminishing $\mathrm{I}^{-}$electronegativity upon increase of $\mathrm{AgI}$ as a result of unpinning. A future version of this model could include a temperature dependence of $S$ for a specific AgI-oxyanion glass composition with a view to account for the $\sigma(T)$ behaviour across the glass transition-an important conductivity relaxation problem. A more ambitious task could be to look at the conductivity phase transition in $\mathrm{AgI}$ and its ternaries. Perhaps at the most fundamental level $S$ serves as a sensitive thermometer of Coulomb interactions in fast-ion conducting glasses.

Aniya (2000a) has examined ionic conduction of superionic glasses in the average electronegativity picture. When a glass $\left(\mathrm{AgI}-\mathrm{Ag}_{2} \mathrm{O}-\mathrm{B}_{2} \mathrm{O}_{3}\right)$ is formed, there is a scrambling of the individual chemical bonds (e.g. Ag-I, $\mathrm{Ag}-\mathrm{O}$ and $\mathrm{B}-\mathrm{O}$ bonds) to produce on the average an electronegative $\chi_{m}$ related to the electronegativity of the glass constituents of the glass as

$$
\chi_{m}=\left(\chi_{\mathrm{A}}^{x} \chi_{\mathrm{B}}{ }^{y} \chi_{\mathrm{C}}^{z} \ldots\right)^{1 /(x+y+z+\ldots)},
$$

where $x, y$ and $z$ are the compositions of A, B and C, respectively.

Ionic conductivity of a number of glasses in the AgI$\mathrm{Ag}_{2} \mathrm{O}-\mathrm{GF}$ (where $\mathrm{GF}=\mathrm{MoO}_{3}, \mathrm{WO}_{3}, \mathrm{P}_{2} \mathrm{O}_{5}, \mathrm{GeO}_{2}$ etc) system has a striking $\mathrm{S}$-shaped correlation with the inverse of electronegativity defined above. Glass network expands upon introducing $\mathrm{AgI}$ to the binary glass $\mathrm{Ag}_{2} \mathrm{O}-$ GF system. If $V_{\mathrm{m}}$ is the molar volume of GF in the binary 
glass and $V_{\mathrm{d}}$ the molar volume in the doped glass, then the fractional volume expansion of the network is $\Delta V_{\mathrm{f}}=\left(V_{\mathrm{d}}-V_{\mathrm{m}}\right) / V_{\mathrm{m}}$. Since $\chi_{\mathrm{m}}$ changes with doping, it is naturally correlated to $\Delta V_{\mathrm{f}} \cdot \chi_{\mathrm{m}}$ vs $\Delta V_{\mathrm{m}}$ plots for different systems for a family that collapses into a single plot when $\chi_{\mathrm{m}}$ is written as $\left(\chi_{\mathrm{m}}+\chi_{0}\right)$ where $\chi_{0}$ is a unique parameter characteristic of a given glass system or in other words a given glass former. Thus it is seen that average electronegativity concept is informative in that it distinguishes between various glass formers and their quantitative role-through $\chi_{0}$-in influencing ion transport in superionic glasses.

\section{Polymer electrolytes}

Ionic conductivity could be induced into insulating polymers by dissolving ionic salts in them. For example, the polymer could be polyethylene oxide or polyvinyl alcohol and the ion could be $\mathrm{Li}^{+}$or $\mathrm{Na}^{+}$in the form of $\mathrm{LiClO}_{4}$ or $\mathrm{NaCl}$ or $\mathrm{H}^{+}$in the form of $\mathrm{NH}_{4} \mathrm{SCN}$. Polymer electrolytes have unique properties: thin film forming ability, good processability, flexibility, lightweight, elasticity/ plasticity, transparency and relatively high ionic conductivity. The polymer host provides easy pathways for the diffusion of ions. These polymers can be amorphous or crystalline-but both forms exhibit ionic conductivity. Generally both forms are present in the sample. This biphasic nature influences conductivity and crystallinity. Ions move in a dynamic environment created by the polymer chain in the amorphous phase above the glass transition temperature, $T_{\mathrm{g}}$ (usually around ambient or slightly above). A crankshaft-like motion associated with short segments of the polymer chain creates randomly suitable coordination sites adjacent to ions so that these ions may hop. Such segmental modes involve the motion of groups of atoms on the polymer chains and are essentially slow, relatively speaking. This slow segmental motion limits hopping rate and maximum conductivity attainable to $\sim 10^{-4} \mathrm{~S} / \mathrm{cm}$ at room temperature. These electrolytes are essentially composites and the conductivity is influenced by the thermally induced morphological changes in the polymer component such as glass transition and crystallization of uncrystallized regions. The conductivity of a typical polymer electrolyte follows Arrhenius type law,

$$
\sigma=\left(\sigma_{0} / T\right) \exp \left(-E_{\mathrm{a}} / k T\right) .
$$

The pre-exponent $\sigma_{0}$ is expressed as

$$
\sigma_{0}=N v_{0} \exp \left(S_{\mathrm{a}} / k\right)
$$

where $N$ is the mobile ion concentration, $v_{0}$ the vibrational frequency of the mobile ions in equilibrium and $S_{\text {a }}$ the entropy of activation for ionic motion. Entropy is involved in charge carrier creation and migration so, $S_{\mathrm{a}}=S_{\mathrm{c}}+S_{\mathrm{m}} \cdot S_{\mathrm{a}}$ and $E_{\mathrm{a}}$ are related by

$$
S_{\mathrm{a}}=E_{\mathrm{a}} / T_{\mathrm{D}},
$$

where $T_{\mathrm{D}}$ is the order-disorder transition temperature in the mobile ion sublattice. Thus the conductivity is given by

$$
\sigma T=N v_{0} \exp \left(E_{\mathrm{a}} / k T\right)\left[\left(1 / T_{\mathrm{D}}\right)-(1 / T)\right],
$$

which may be used to interpret experimental results.

A linear correlation exists between $\sigma_{0}$ and $E_{\mathrm{a}}$ known as Meyer-Neldel rule or compensation law:

$$
\ln \sigma_{0}=\alpha E_{\mathrm{a}}+\beta
$$

where $\alpha=1 / k T_{\mathrm{D}}$ and $\beta=\ln \left(N v_{0}\right)$.

Typical conductivity and Meyer-Neldel plots for $15 \%$ $\mathrm{NH}_{4} \mathrm{SCN}$ - PVA composites are shown in figure 9 (Shukla and Agrawal 1998). It turns out that the $T_{\mathrm{D}}$ 's obtained
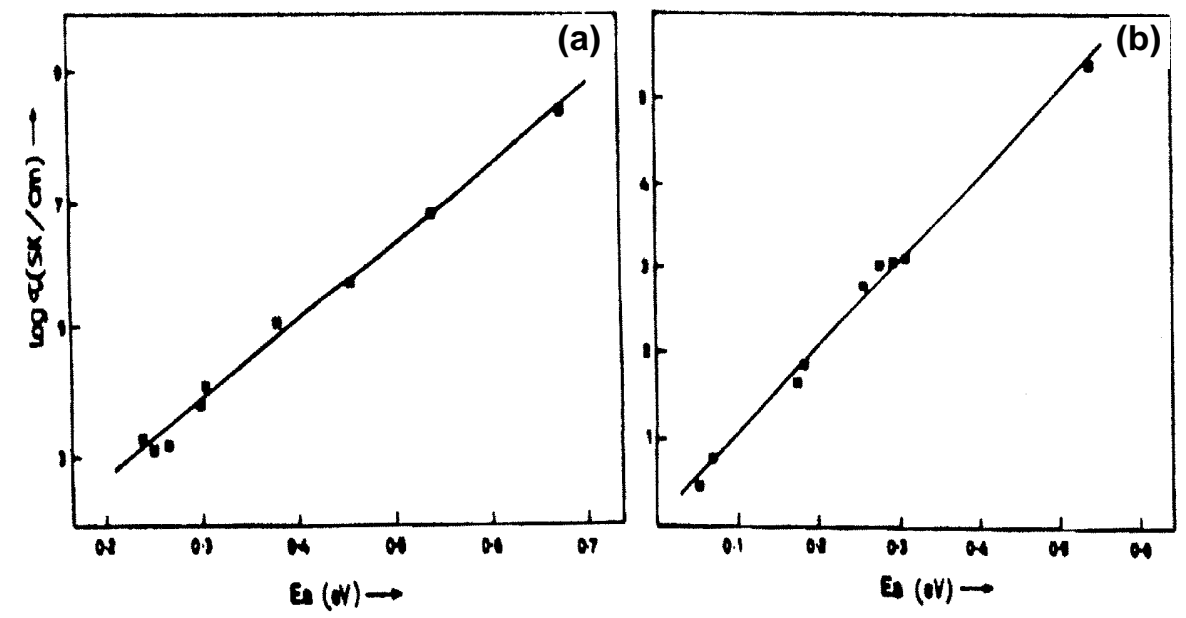

Figure 9. Meyer-Neldel plots for $15 \% \mathrm{NH}_{4} \mathrm{SCN}-\mathrm{PVA}$ composites. (a) Low temperature region $\left(T<T_{\mathrm{g}}\right)$ and (b) high temperature region $\left(T>T_{\mathrm{g}}\right)$ (see text for details). 
from an analysis of the low temperature conductivity data correspond to the glass transition temperature of the composite while those obtained from an analysis of high temperature data corresponds to the melting point of the crystalline PVA phase. Migration entropy appears to play an important role in the optimization of conductivity at room temperature.

$\mathrm{LiClO}_{4}$ : PEO is an interesting $\mathrm{Li}^{+}$ion conducting system (Kato et al 1981) in which PEO chains coil (a) $\mathrm{Li}^{+}$ cations and isolate them from $\mathrm{ClO}_{4}^{-}$, (b) the transport number of $\mathrm{Li}^{+}$, defined as the ratio, $\mu^{+} /\left(\mu^{+}+\mu^{-}\right)$, where $\mu^{+}$ and $\mu^{-}$are the mobilities of cationic and anionic species, is 0.5 which implies that both type of ions are mobile. This is unusual for a $\mathrm{Li}^{+}$ion conductor, (c) $\mathrm{Li}^{+}$ion migrates with the segmental motion of the polymer, which indicates a strong ion-dipole interaction leading to solvation by ethylene oxide and (d) $\mathrm{Li}^{+}$transport number is influenced by the $\mathrm{LiClO}_{4}$ content or $T_{\mathrm{g}}$, the PEO glass transition temperature of the polymer.

The observation (b) can be generalized as follows: Under the same energy level of the segmental motion of matrix polymers, carrier ions which have a large radius, can move faster in that matrix. This is because the larger ion has a weaker interaction force through the ion-dipole interaction. This important theoretical idea offers two possibilities for the design of polymer electrolytes.

(i) Choose an ion with a large radius, say $\mathrm{Rb}^{+}$instead of one with small radius such as $\mathrm{Li}^{+}$or

(ii) Design a polymer matrix, which has weaker iondipole interaction to the mobile ions.

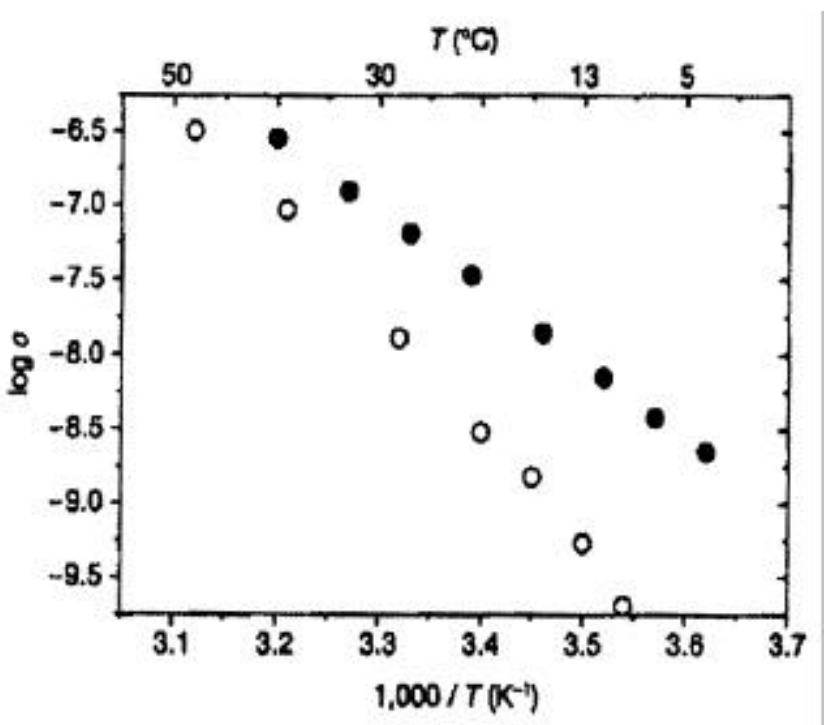

Figure 10. Ionic conductivity $(\sigma, \mathrm{S} / \mathrm{cm})$ of amorphous (open circles) and crystalline (close circles) $\mathrm{PEO}_{6}-\mathrm{LiSbF}_{6}$ as a function of [temperature $]^{-1}$. The crystalline polymer electrolyte has larger conductivity than its amorphous counterpart throughout the temperature range studied (Angell 1993).
Generally speaking, polymer based systems with a low $T_{\mathrm{g}}$ are likely to be better fast ion conductors. Recent new approaches to the design of polymers with high conductivity include 'rubbery' solid electrolytes (Angell 1993): use of only small amounts of high molecular weight polymers bestows 'rubbery' properties by means of the 'entanglement' mechanism. Decoupled cation motion with high conductivity and rubbery compliance is obtainable subject to the following four conditions being met.

High salt solution must cool without salt crystallization yet retain high conductivity, and have sub ambient $T_{\mathrm{g}}$ to provide rubbery but not glassy compliance when polymer is added. Solubility of small amount of polymer in salt melt must result in rubbery compliance but with no excessive reduction of conductivity. Solution with the above properties must be a simple ion conductor. Electrolyte must be reasonably stable electrochemically.

Preliminary studies on polymer electrolytes with as little as 10 parts polypropylene oxide in a mixture of LiI, Li-acetate and $\mathrm{LiClO}_{4}(50: 30: 20)$ have shown that it is possible to meet the abovesaid conditions. $\mathrm{Li}^{+}$does dominate conductivity in these systems with $T / T_{\mathrm{g}}=1 \cdot 2$ $\left(25<T<50^{\circ} \mathrm{C}\right)$. The decoupling index $R$ is $\sim 10^{2}$ corresponding to a transport number of 0.9 .

Another strategy (Gadjourova et al 2001) is to emphasize order and structure and seek new crystalline polymer electrolytes with suitable structure and with partial occupancy of sites by potentially mobile ions. The selectivity of $\mathrm{Li}^{+}\left(t_{+}=1\right)$ is exploited here.

$\mathrm{PEO}_{6}$ is a polymer $(\mathrm{MW}=10,000)$ that meets the above design conditions. This crystalline polymer into which $\mathrm{LiSbF}_{6}$ has been incorporated possesses conductivity higher than that of its amorphous counterpart. This is possible because structural sites are present in the crystalline polymer into which vibrating ions with sufficient energy to help can migrate. Permanent pathways for ion transport of the type present in AgI or its ternary versions, but in a static environment, are available in these materials.

Mechanically strong polymer electrolytes have been built (Croce et al 1998) by adding nanometer sized (5.8$13 \mathrm{~nm}) \mathrm{TiO}_{2}$ and $\mathrm{Al}_{2} \mathrm{O}_{3}$ powders to $\mathrm{PEO}: \mathrm{LiClO}_{4}$ to make nanocomposite polymer electrolytes. The $\mathrm{TiO}_{2}$ or $\mathrm{Al}_{2} \mathrm{O}_{3}$ powder acts as a solid plasticizer for PEO and kinetically inhibits crystallization on annealing from amorphous state above $60^{\circ} \mathrm{C}$. The low temperature conductivity $\left(\sim 10^{-5} \mathrm{~S} / \mathrm{cm}\right.$ at $30^{\circ} \mathrm{C}$ for $\mathrm{TiO}_{2}$ added electrolyte) is promoted by the high surface area of the dispersed filler while the network of the dispersed filler induces improved mechanical stability. The ceramic grains may act as cross-linking centres for PEO segments thereby lowering the reorganizing tendency of the polymer chain and promoting preferred $\mathrm{Li}^{+}$transport at the boundaries of the ceramic particles. A heterogeneous polymer electrolyte is thus realized.

As polymers have subambient glass transition temperatures, there is a continuous motion of segments of the 
polymer macromolecule at ambient and above. This dynamic disorder is believed to assist ion transport in polymer electrolytes. A comprehensive theory of ion transport in polymers is not yet available.

The theoretical explanation of the dependence of ionic conductivity on temperature, salt fraction and frequency is complicated by the multiphasic (several crystalline and amorphous phases) nature and processing and thermal history dependence of these phases.

The salt fraction induced variation of ionic conductivity is an interesting aspect of this theoretical effort because variation of salt fraction changes (a) the ratio of crystalline to amorphous regions and (b) the charge carrier concentration supplied by the salt. A final goal can be to predict the ionic conductivity of an unknown polymer electrolyte for a given salt fraction in the search for a high conducting polymer electrolyte. Conductivity in disordered media is usually studied computationally by considering the continuous time random walk of an ion in the medium. The random walk can be 'accelerated' by making the walker jump at every step but making it adjust the time to account for the waiting time.

A dynamic bond percolation model (Tarafdar 2001) computes conductivity of a polymer by simulating a hopping model. A renewal time characterizes the dynamic nature of the medium. This model explains the frequency dependence of conductivity within a range of not so high frequencies. In what may be called a 'dynamic phase reorganization' model, a regular lattice represents the polymer where sites represent either crystalline or amorphous regions, with the mobile ions performing a random walk. The walk algorithm takes care of the reorganization of the different phases over time scale comparable to the conduction process. This model provides a qualitative explanation for the conductivity dependence of salt concentration for $\mathrm{PEO}-\mathrm{NH}_{4} \mathrm{I}$ and $\mathrm{PEO}-\mathrm{NH}_{4} \mathrm{SCN}$ electrolytes. It also estimates average jump distances that are consistent with the experimental bond lengths in these polymers.

The conductivity of PEO- $\mathrm{NH}_{4} \mathrm{ClO}_{4}$ films for weight fractions of salt in the range $x=0-0.35$ show interesting correlation (Bhattacharya et al 1999) with crystallinity and morphology. Crystallinity decreases and reaches a minimum for $x=0.18$ as the salt fraction increases and the minimum in $x$ corresponds to a maximum in conductivity $\sigma$ thus showing an inverse correlation with crystallinity. This maximum in $\sigma$ corresponds to a crossover from diffusion-limited aggregation or fractal formation regime to regime in which compact polygonal spherulites are formed. As these spherulites grow to their largest size, a sharp fall in $\sigma$ occurs.

Large size $(\sim 2-3 \mathrm{~cm})$ fractal formation observed in PEO-NH ${ }_{4}$ I films (Chandra and Chandra 1994) has been attributed to a random walk and subsequent aggregation of iodine removed from the polymer complex with $\mathrm{Al}_{2} \mathrm{O}_{3}$ aiding this process. These iodine fractals introduce grain boundaries and regions of electronic conduction, which could be separated by impedance spectroscopy.

Ionic conduction in the fractal regime takes place through the amorphous regions in the interstices of the fractal aggregates. In the spherulitic region, dynamic disorder takes over and ion motion is described by segmental motion of polymer network.

\section{Conclusions}

Some of the important theoretical approaches to the understanding of superionic conductivity in (poly) crystalline, glassy and polymeric materials have been reviewed briefly. The following conclusions emerge:

(I) Phase transition to the superionic conducting state in AgI-family is apparently triggered by cluster formation and strong mobile-ion interaction within the clusters, which render the low temperature structure unstable. Anomalies in ionic conductivity and related physical properties are successfully explained in the cluster induced distortion model. The weakly covalent Ag-I bond and the open crystal structure are very crucial in the physics of these types of systems.

(II) Ionic composites invariably involve conducting and non conducting phases and the all important interface between the two, whose space charge enhances the conductivity considerably and also trigger phase transitions to exotic polymorphic phases, for which the mechanisms are yet to be explored.

(III) (Super) ionic conductivity in glasses essentially arises from the dynamics of ion hopping. The frequency dependence of conductivity exhibit from unusual power law dependence (non-Debye relaxation) that is explained by mode relaxation theory using the concept of mode diffusion length. A diffusive mode contributes to d.c. conductivity while non-diffusive modes give rise to a.c. conductivity. An attractive concept of mismatch and relaxation formalizes the jump relaxation model of conduction of superionic glasses and establishes the frequency-time domain in the process.

(IV) Theory of ionic conductivity in polymer electrolytes $\left(\mathrm{PEO}: \mathrm{LiClO}_{4}\right)$ is yet in its infancy because of its complex structural nature and the fact that conductivity is intimately connected to preparative and thermal history, composition and crystallinity. Several new approaches to synthesis of optimally useful polymer electrolytes such as 'rubbery' electrolytes, crystalline polymers and nano composites have been explored, which augur well for the future.

\section{Acknowledgement}

The authors thank the referees for their invaluable comments and suggestions. 


\section{References}

Angell C A et al 1993 Nature 362137

Aniya M and Wakamura K 1998 Solid state ionics: Science and technology (eds) B V R Chowdari et al (Singapore: World Scientific) p. 43

Aniya M 2000a Solid State Ionics 136-137 1085

Aniya M 2000b Solid state ionics: Materials and devices (eds) B V R Chowdari and W Wang (Singapore: World Scientific) p. 51

Bhattacharya A J et al 1997 Solid State Ionics 95283

Bhattacharya A J et al 1999 Phys. Rev. B60 909

Chandra A and Chandra S 1994 Phys. Rev. B49 633 and references therein

Croce F et al 1998 Nature 394456

Funke K 1997 Defects and Diffusion Forum 143-147 1243

Funke K and Wilmer D 2000 Solid State Ionics 136-137 1329 and references therein

Funke K et al 1996 Solid State Ionics 85293

Funke K et al 1997 Solid State Ionics 9427

Gadjourova Z et al 2001 Nature 412520

Govindaraj G and Murugaraj R 2000 Mater. Sci. and Eng. B77 60

Hairetdinov E F et al 1994 Phys. Rev. B50 13259

Ingram M D 1998 Solid state ionics: Science and technology (eds) B V R Chowdari et al (Singapore: World Scientific) p. 21

Ishii T and Kamishima O 1999 J. Phys. Soc. Jpn 683580

Ishii T and Abe T 1999 J. Phys. Soc. Jpn 683127 and references therein

Jain H 1999 Metal Mater. \& Process. 11317

Kato Y et al 1981 Solid State Ionics 40/41 632

Kawamura J and Shimoji M 1986 J. Non-Cryst. Solids 88295

Kvist A and Josefson A M 1968 Z. Naturforsch. 23a 625

Laskar A L 1992 Solid state ionics (eds) S Chandra et al (Singapore: World Scientific) p. 459

Lee J S et al 2000 J. Electrochem. Soc. 1472407 and references therein

Lidiard A B 1957 Handb. Phys. 20246

Maass P et al 1996 Phys. Rev. Lett. 771528

Maier J 1985 Mater. Res. Bull. 20383

Maier J 2000 Z. Anorg. Allg. Chem. 426264

Ngai K L 1980 Com. Solid State Phys. 9141

Nolting J and Rein D 1969 Z. Phys. Chem. Neue Folge 66150

Ogawa H and Kobayashi M 2002 Solid State Ionics 148211

Rao K J 2002 Structural chemistry of glasses (Amsterdam: Elsevier)

Patnaik J R G and Sunandana C S 1998 J. Phys. Chem. Solids 591059

Salamon M B 1975 Physics of superionic conductors (Berlin: Springer)

Senthil Kumar P, Kini N S, Umarji A M and Sunandana C S 2000 in Solid state ionics: Materials and devices (eds) B V R Chowdari and W Wang (Singapore: World Scientific) pp 121-124

Shastry M C R and Rao K J 1989 Solid State Ionics 3717

Shukla P K and Agrawal S L 1998 Solid state ionics: Science and technology (eds) B V R Chowdari et al (Singapore: World Scientific) p. 211

Shukla A K and Sharma V 1992 Solid state ionics (eds) S Chandra et al (Singapore: World Scientific) p. 91 and references therein

Suresh Chandra 1981 Superionic solids: Principles and applications (Amsterdam: North Holland)

Sunandana C S 1995 Bull. Mater. Sci. 1817

Sunandana C S 1997 J. Phys. Chem. Solids 581359

Sunandana C S 1998 Bull. Mater. Sci. 211

Sunandana C S and Senthil Kumar P 2003 (in preparation)

Takahashi H 1987 J. Phys. Soc. Jpn 562520

Tarafdar S 2001 Ion conducting materials (eds) A R Kulkarni and P Gopalan (New Delhi: Narosa) p. 1

Uvarov N F 2000 Solid state ionics 136-137 1267

Vidyullatha M and Sunandana C S 1993 Solid State Commun. 88553

\section{Note added in proof}

Two very interesting and relevant articles-both dealing with polymer electrolytes-have appeared recently. O Durr et al (2002 Solid State Ionics 149 125) have sought a semi-microscopic description of ion transport in PEOtype polymer electrolytes, on the basis of discrete stochastic moves of individual molecular units. Diffusion coefficients for ions and centre of mass motions have been computed by Monte Carlo simulation as functions of model parameters, also incorporating pressure effects. In an even more coarse-grained description they have considered diffusion in an athermal system of point-like particles (ions) and chains (polymer), thereby providing a quantitative verification of the concept of dynamic percolation. They have obtained diffusion constants for chains and ions and these show VTF behaviour. The VTF temperature is found to increase with ion content $(x)$ in the polymer. For large $x$, the ion and chain motions decouple. Ion diffusion coefficients decrease exponentially with increasing chain content.

Th J Singh and S V Bhat (2003 Bull. Mater. Sci. 26 707) have reported a new solid polymer electrolyte $(\mathrm{PEG})_{x} \mathrm{LiClO}_{4}$ (mol. wt. $\left.=2000\right)$. The maximum electrical conductivity of $0.703 \mu \mathrm{S} / \mathrm{cm}$ obtained for $x=46$ at room temperature reaches a high value of $\sim 100 \mu \mathrm{S} / \mathrm{cm}$ close to the melting point $\left(52 \cdot 3^{\circ} \mathrm{C}\right)$. The temperaturedependence of conductivity shows a crossover from a low-temperature Vogel-Tamman-Fulcher behaviour to a high-temperature Arrhenius behaviour at a characteristic temperature. ${ }^{7} \mathrm{Li}$ NMR shows the highest mobility for this composition. It would be interesting to derive viscosities $(\eta)$ from NMR diffusion constants via Stokes-Einstein equation and then use VTF equation to analyse the temperature dependence of $\eta$. 